\title{
Direction-of-Arrival Tracking Scheme for DS/CDMA Systems: Direction Lock Loop
}

\author{
Seunghyun Min, Student Member, IEEE, Dongyoun Seo, Kwang Bok Lee, Member, IEEE, \\ Hyuck M. Kwon, Senior Member, IEEE, and Yong-Hwan Lee, Member, IEEE
}

\begin{abstract}
In this paper, a new direction-of-arrival (DOA) tracking algorithm, direction lock loop (DiLL), is proposed for wireless direct-sequence code-division multiple-access systems. It has a similar concept to the delay lock loop that is used for timing synchronization. It may track the DOA of sources by iterations. Its computational requirements are $N K+\mathrm{O}(K)$ for coherent DiLL and $2 N K+\mathrm{O}(K)$ for noncoherent DiLL, where $N$ is the number of antenna elements and $K$ is the number of signal sources, which is less than that of the projection approximation subspace tracking with deflation algorithm [5]. The DOA tracking accuracy is demonstrated by analysis and computer simulations.
\end{abstract}

Index Terms - Array response vector, direction of arrival (DOA), direction lock loop (DiLL), DOA tracking, direct-sequence codedivision multiple access (DS/CDMA), smart antenna.

\section{INTRODUCTION}

$\mathbf{T}$ HE demand for wireless communication services is growing at an explosive rate. To enhance the capacity of wireless communication systems, space-division multiple-access (SDMA) systems are implemented by using smart antenna systems and spatial filtering interference reduction (SFIR). In recent years, smart antenna systems based on direction-of-arrival (DOA) have been developed to effectively suppress undesired signals from other directions by forming a beam pattern [1].

In the smart antenna systems, the problem of estimating the DOA of multiple moving sources is of great interest and many DOA estimation techniques have been developed. Eigen structure methods such as MUSIC and ESPRIT have been widely used in providing estimates of the DOA of signals [2], [3]. These algorithms are developed to exploit the benefits of temporal integration of the array data, thus usually have deteriorated performance in the presence of moving sources because they provide reduced resolution due to the spreading of the array spatial

Manuscript received March 26, 2002; revised October 15, 2002; accepted January 13,2003. The editor coordinating the review of this paper and approving it for publication is M. Sawahashi.

S. Min was with the School of Electrical Engineering and INMC, Seoul National University, Seoul 151-742, Korea. He is now with the Telecommunication System Division, Samsung Electronics, Gyeonggi-do 442-600, Korea (e-mail:seunghyun.min@samsung.com).

K. B. Lee and Y.-H. Lee are with the School of Electrical Engineering and INMC, Seoul National University, Seoul 151-742, Korea (e-mail: klee@snu.ac.kr).

D. Seo is with the Standardization and System Research Group, LG Electronics, Kyongki-do 431-739, Korea.

H. M. Kwon is with the Department of Electrical and Computer Engineering, Wichita State University, Wichita, KS 67260-0044 USA.

Digital Object Identifier 10.1109/TWC.2003.821215 spectrum caused by the motion of the sources [4]. The computational burden of the eigen analysis also increases significantly with the number of antenna elements.

To overcome the spread of the spatial spectrum and the computational requirement of the eigen methods, subspace tracking algorithms have been proposed such as projection approximation subspace tracking with deflation algorithm (PASTd) [5]. This algorithm tracks a signal subspace recursively. Generally, it has computational requirements of $4 N K+\mathrm{O}(K)$, where $N$ is the number of antenna elements and $K$ is the number of signal sources. The computational requirement can be reduced to $3 N K+\mathrm{O}(K)$ in direct-sequence code-division multiple-access (DS/CDMA) systems with the assumption that a signal power is much stronger than that of the multiple access interference (MAI) after despreading [6]. Using this algorithm, the eigenvectors may be tracked recursively.

Recently, several approaches have been developed to deal with the problem of estimating the DOA of moving sources by use of an antenna array, including also the parametric tracking algorithms [4], [7]-[14]. A general assumption used in these techniques is that the sources can be approximated as stationary during a limited integration time interval.

In this paper, a DOA tracking scheme is proposed for DS/CDMA systems. Since this scheme is similar to the delay lock loop (DLL) used for code tracking in DS/CDMA systems [15]-[17], it is referred to as the direction lock loop (DiLL) scheme in this paper and similarly in [18]-[20]. In [21], the DLL concept was applied to the beam tracking algorithm for fast moving signal sources. However, it was developed to track not the DOA of signals but the beam pattern. Thus, we cannot track the DOA of signals using the algorithm proposed in [21]. An error signal for the DOA tracking is generated from the spatial correlation of an input signal and the array response vectors whose directions are $\pm \Delta \theta$ shifted from the current DOA tracking value. This error signal is used to iteratively update DOA tracking value. The DiLL may be implemented in both coherent and noncoherent modes. The computational requirements of the two DiLL schemes are $N K+\mathrm{O}(K)$ for coherent mode and $2 N K+\mathrm{O}(K)$ for noncoherent mode, which is less than that of PASTd algorithm. However, the DiLL scheme cannot track properly, when the desired signal incidents from two or more directions within one chip duration.

The DiLL scheme will operate properly when the initial DOA tracking value is within a certain range which is referred to as the locking range like the DLL scheme in timing synchronization. Thus, the initial DOA tracking scheme is 


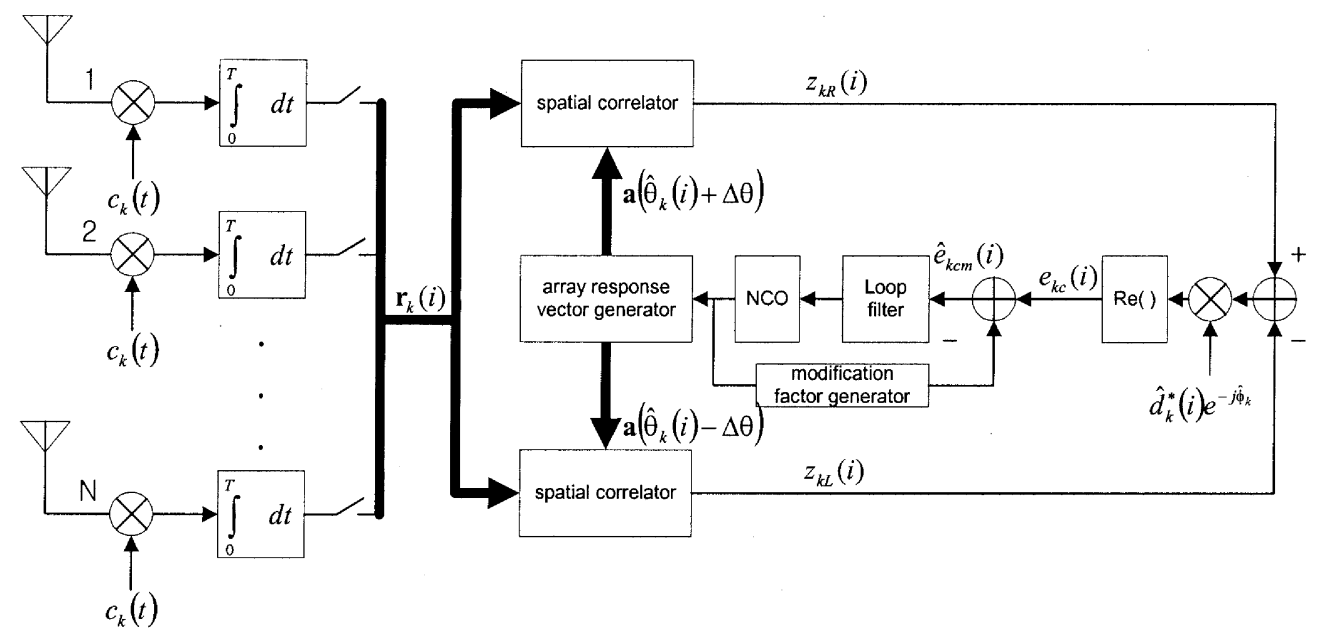

Fig. 1. Block diagram of the coherent DiLL scheme.

required for the DiLL scheme which is similar to the DLL scheme that is proceeded by the acquisition in the timing synchronization for DS/CDMA systems. In this paper, the initial DOA tracking scheme is also proposed by modifying the DiLL scheme.

This paper is organized as follows. The system model is shown in Section II. In Section III, the new DOA tracking scheme, coherent DiLL is presented and the characteristics of the coherent DiLL scheme are explained. The noncoherent DiLL is then presented in Section IV. The initial tracking stage of the DiLL scheme is discussed in Section V. In Section VI, the performance analysis and the numerical results are shown. Conclusions are drawn in Section VII.

\section{SySTEM MODEL}

Consider a uniform linear array with $N$ half-wavelength spaced antenna elements. Thus, the received signal at the $n^{\text {th }}$ antenna may be represented as

$$
r_{n}(t)=\sum_{k=1}^{K} e^{j(n-1) \pi \sin \theta_{k}(t)+\phi_{k}} s_{k}(t)+v_{n}(t)
$$

where $K$ is the number of sources, $\theta_{k}(t)$ is the DOA of the $k$ th sources, $\phi_{k}$ is the carrier phase which is uniformly distributed in $[0,2 \pi], s_{k}(t)=\sum_{i=-\infty}^{\infty} d_{k}(i) c_{k}\left(t-i T-\tau_{k}\right)$, $d_{k}(i)$ is the transmitted data, $c_{k}(t)$ denotes the spreading sequence waveform with processing gain, $L, v_{n}(t)$ is the additive white Gaussian noise, $\tau_{k}$ and $T$ is the time delay and the symbol interval, respectively. We may write the received signals in vector form

$$
\mathbf{r}(t)=\mathbf{A}(\boldsymbol{\theta}(t)) \mathbf{S}(t)+\mathbf{v}(t)
$$

where

- $\mathbf{r}(t)=\left[\begin{array}{llll}r_{1}(t) & r_{2}(t) & \cdots & r_{N}(t)\end{array}\right]^{\mathrm{T}}$ is an $N \times 1$ vector of received signals at time $t$ where the superscript $\mathrm{T}$ denotes the vector transpose;
- $\boldsymbol{\theta}(t)=\left[\begin{array}{llll}\theta_{1}(t) & \theta_{2}(t) & \cdots & \theta_{K}(t)\end{array}\right]^{\mathrm{T}}$ is the source DOA parameter vector;

- $\mathbf{A}(\boldsymbol{\theta}(t))$ is the composite array response matrix which is determined by the DOA of signals. The $k^{\text {th }}$ column of $\mathbf{A}(\boldsymbol{\theta}(t))$; is defined as the array response vector associated with the $k^{\text {th }}$ source and is given by $\mathbf{a}\left(\theta_{k}(t)\right)=$ $\left[\begin{array}{llll}e^{j 0} & e^{-j \pi \sin \theta_{k}(t)} & \cdots & e^{-j(N-1) \pi \sin \theta_{k}(t)}\end{array}\right]^{\mathrm{T}}$

- $\mathbf{S}(t)=\operatorname{diag}\left[s_{1}(t) e^{j \phi_{1}} \quad s_{2}(t) e^{j \phi_{2}} \quad \cdots \quad s_{K}(t) e^{j \phi_{K}}\right]$;

- $\mathbf{v}(t)=\left[v_{1}(t) v_{2}(t) \cdots v_{N}(t)\right]^{\mathrm{T}}$ is an $N \times 1$ additive noise vector, which is assumed to be spatially and temporally white Gaussian.

\section{COHERENT DiLL}

In this section, a DOA tracking scheme, coherent DiLL, is presented for DS/CDMA systems.

\section{A. Principle of the Coherent DiLL Scheme}

In this section, the principle of the coherent DiLL scheme is explained. Fig. 1 shows a block diagram of the coherent DiLL scheme. Note that its structure is similar to the DLL for code tracking in DS/CDMA systems [15]-[17]. The received signals are despread by the users' own spreading sequences for each of the antenna elements. Therefore, the $k^{\text {th }}$ user's despread and sampled array vector signal, $\mathbf{r}_{k}(i)$ may be represented as

$\mathbf{r}_{k}(i)=d_{k}(i) \mathbf{a}\left(\theta_{k}(i)\right) e^{j \phi_{k}}+\sum_{\substack{l=1 \\ l \neq k}}^{K} \psi_{k l} d_{l}(i) \mathbf{a}\left(\theta_{l}(i)\right) e^{j \phi_{l}}+\mathbf{v}_{k}(i)$

where the cross correlation of the spreading sequences, $\psi_{k l}$ may be defined as $\psi_{k l} \triangleq \int_{0}^{\mathrm{T}} c_{k}(t) c_{l}(t) d t$ when the time delay of all users are zero and $\mathbf{v}_{k}(i)$ is the despread noise signal vector whose covariance matrix is $\sigma^{2} \mathbf{I} . \mathbf{r}_{k}(i)$ is spatially correlated simultaneously with right-shifted and left-shifted array response vectors $\mathbf{a}\left(\hat{\theta}_{k}(i)+\Delta \theta\right)$ and $\mathbf{a}\left(\hat{\theta}_{k}(i)-\Delta \theta\right)$ to produce correlator outputs $z_{k R}(i)$ and $z_{k L}(i)$, when the DOA tracking value at the $i^{\text {th }}$ time is $\hat{\theta}_{k}(i)$ and the shift angle is $\Delta \theta$ which is set to a 
constant value. The spatial correlator outputs $z_{k R}(i)$ and $z_{k L}(i)$ may be represented as

$$
\begin{aligned}
z_{k R}(i)= & \frac{1}{N} \mathbf{a}^{H}\left(\hat{\theta}_{k}(i)+\Delta \theta\right) \mathbf{r}_{k}(i) \\
= & d_{k}(i) R\left(\theta_{k}(i), \hat{\theta}_{k}(i)+\Delta \theta\right) e^{j \phi_{k}} \\
& +I_{k R}(i)+v_{k R}(i) \\
z_{k L}(i)= & \frac{1}{N} \mathbf{a}^{H}\left(\hat{\theta}_{k}(i)-\Delta \theta\right) \mathbf{r}_{k}(i) \\
= & d_{k}(i) R\left(\theta_{k}(i), \hat{\theta}_{k}(i)-\Delta \theta\right) e^{j \phi_{k}} \\
& +I_{k L}(i)+v_{k L}(i)
\end{aligned}
$$

where $R\left(\theta_{1}, \theta_{2}\right)$ is a normalized spatial correlation function, which is defined as

$R\left(\theta_{1}, \theta_{2}\right)=\frac{1}{N} \mathbf{a}^{H}\left(\theta_{2}\right) \mathbf{a}\left(\theta_{1}\right)=\frac{1}{N} \sum_{n=1}^{N} e^{-j \pi(n-1)\left(\sin \theta_{1}-\sin \theta_{2}\right)}$

$I_{k R}=\sum_{\substack{l=1 \\ l \neq k}}^{K} \psi_{k l} d_{l}(i) R\left(\theta_{l}(i) \hat{\theta}_{k}(i)+\Delta \theta\right) e^{j \phi_{l}}$ and $I_{k L}=\sum_{\substack{l=1 \\ l \neq k}}^{K} \psi_{k l} d_{l}(i) R\left(\theta_{l}(i), \hat{\theta}_{k}(i)-\Delta \theta\right) e^{j \phi_{l}}$ are MAI terms, and $v_{k R}(i)=(1 / N) \mathbf{a}^{H}\left(\theta_{k}(i)+\Delta \theta\right) \mathbf{v}(i)$ and $v_{k L}(i)=(1 / N) \mathbf{a}^{H}\left(\theta_{k}(i)-\Delta \theta\right) \mathbf{v}_{k}(i)$ are the noise terms in $z_{k R}(i)$ and $z_{k L}(i)$, respectively. The superscript $H$ denotes the Hermitian transpose. Under the assumption that the desired signal power is large enough compared to each of the other user signals after the despreading operation, MAI terms may be treated as background additive white Gaussian noise (AWGN).

The difference between the two correlator outputs is multiplied by a conjugate of transmitted data estimate $\hat{d}_{k}^{*}(i)$ and carrier phase estimate $e^{-j \hat{\phi}_{k}}$ to remove the effect of $d_{k}(i)$ and $\phi_{k}$, and then we take the real part to produce an error signal $e_{k c}(i)$ in the coherent mode, which is defined as

$$
e_{k c}(i)=\operatorname{Re}\left[\left(z_{k R}(i)-z_{k L}(i)\right) \hat{d}_{k}^{*} e^{-j \hat{\phi}_{k}}\right]
$$

where $*$ denotes the complex conjugate and the subscript $c$ denotes a coherent mode. With the assumption of $\hat{d}_{k}(i)=d_{k}(i)$ and $\hat{\phi}_{k}=\phi_{k}$, (6) may be represented as

$$
e_{k c}(i)=G_{c}\left(\hat{\theta}_{k}(i) \mid \theta_{k}(i)\right)+w_{k c}(i)
$$

where $G_{c}\left(\hat{\theta}_{k}(i) \mid \theta_{k}(i)\right)$ is defined as $\operatorname{Re}\left[R\left(\theta_{k}(i), \hat{\theta}_{k}(i)+\right.\right.$ $\left.\Delta \theta)-R\left(\theta_{k}(i), \hat{\theta}_{k}(i)-\Delta \theta\right)\right], w_{k c}(i)$ is defined as $\operatorname{Re}\left[\left(I_{k R}(i)-I_{k L}(i)+v_{k R}(i)-v_{k L}(i)\right) d_{k}^{*} e^{-j \phi_{k}}\right] . \quad$ The plot of $G_{c}\left(\hat{\theta}_{k}(i) \mid \theta_{k}(i)\right)$ is referred to as the S-curve because the shape of $G_{c}\left(\hat{\theta}_{k}(i) \mid \theta_{k}(i)\right)$ is similar to the S-curve in the DLL. The error signal, $e_{k c}(i)$ is filtered and fed to the numerically controlled oscillator (NCO) to update DOA tracking value iteratively. Hence, the DOA tracking value at the $(i+1)^{\text {th }}$ time may be expressed as

$$
\hat{\theta}_{k}(i+1)=\hat{\theta}_{k}(i)+K_{0} \cdot\left(e_{k c}(i) \otimes f(i)\right)
$$

where $f(i)$ is the impulse response of the loop filter, $K_{0}$ is the $\mathrm{NCO}$ gain, and $\otimes$ denotes convolution.

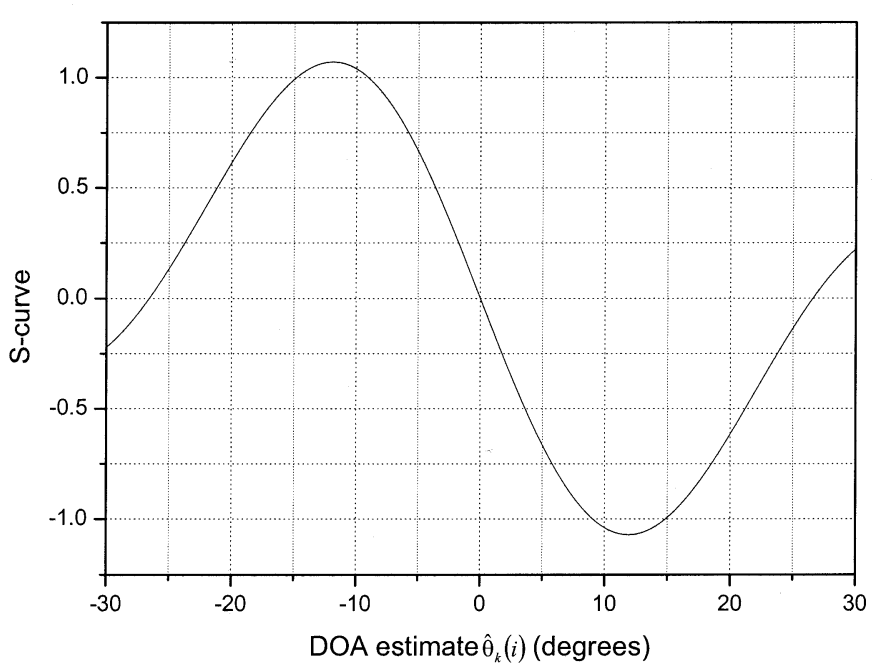

(a)

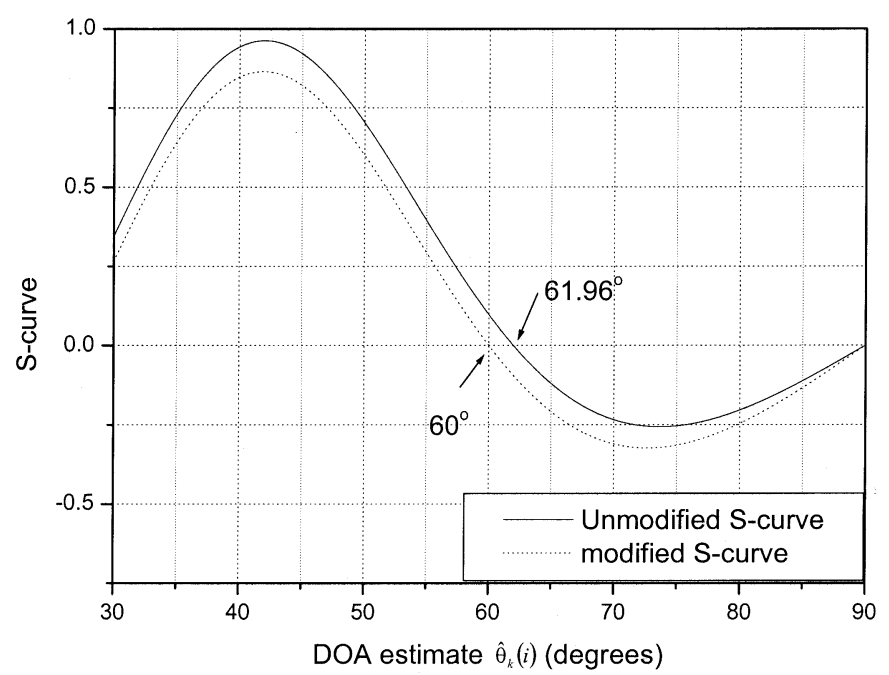

(b)

Fig. 2. S-curve. (a) S-curve $\left(N=4, \Delta \theta=11.14^{\circ}, \theta_{k}(i)=0^{\circ}\right)$. (b) Modified and unmodified S-curve $\left(N=4, \Delta \theta=11.14^{\circ}, \theta_{k}(i)=60^{\circ}\right)$.

Fig. 2(a) shows the plot of $G_{c}\left(\hat{\theta}_{k}(i) \mid \theta_{k}(i)\right)$ as a function of $\hat{\theta}_{k}(i)$, when $\theta_{k}(i)=0^{\circ}, N=4$, and $\Delta \theta=11.14^{\circ}$. When the DOA tracking value $\hat{\theta}_{k}(i)$ at the $i^{\text {th }}$ time is less than the actual DOA $\theta_{k}(i)$, the error signal is larger than zero and, thus. NCO increases the DOA tracking value at the $(i+1)^{\text {th }}$ time. When $\hat{\theta}_{k}(i)$ is larger than $\theta_{k}(i)$, the error signal is less than zero, and thus NCO decreases $\hat{\theta}_{k}(i+1)$. Repeating this procedure, $\hat{\theta}_{k}(i)$ converges to the DOA of the signal $\theta_{k}(i)$. Thus, the DiLL scheme tracks the DOA of moving sources iteratively.

\section{B. Modification Factor}

In the DiLL scheme, to track the DOA of a signal source correctly, the value of the S-curve should be zero when $\hat{\theta}_{k}(i)$ is $\theta_{k}(i)$. As shown in Fig. 2(a), when $\theta_{k}(i)$ is $0^{\circ}$, the value of the S-curve is zero. This ensures that $\hat{\theta}_{k}(k)$ converges to $0^{\circ}$. However, the value of the S-curve is not always zero when $\hat{\theta}_{k}(i)$ is $\theta_{k}(i)$. Fig. 2(b) shows the S-curve for $\theta_{k}(i)$ is $60^{\circ}, N$ is 4 and $\Delta \theta$ is $11.14^{\circ}$, and indicates that $\hat{\theta}_{k}(i)$ does not approach to $60^{\circ}$ but will instead converge 
to $61.96^{\circ}$. This means that the coherent DiLL scheme is a biased estimator. $G_{c}\left(\theta_{k}(i) \mid \theta_{k}(i)\right)$ may be represented as (9), shown at the bottom of the next page. The term in (9), $\left(\sin \left(\theta_{k}(i)\right)-\sin \left(\theta_{k}(i)-\Delta \theta\right)\right)-\left(\sin \left(\theta_{k}(i)+\Delta \theta\right)-\sin \left(\theta_{k}(i)\right)\right)$, is not generally zero due to the nonlinearity of the sine function. This is why $G_{c}\left(\theta_{k}(i) \mid \theta_{k}(i)\right)$ is not generally zero. From (9), $G_{c}\left(\theta_{k}(i) \mid \theta_{k}(i)\right)$ is found to increase, as $\Delta \theta$ increases.

To remove the bias, the error signal should be modified as follows. For simplification of representation, $G_{c}\left(\theta_{k}(i) \mid \theta_{k}(i)\right)$ is denoted by $m_{c}\left(\theta_{k}(i)\right)$, referred to as a modification factor. The modified error signal $e_{k c m}(i)$ is generated by subtracting the modification factor from $e_{k c}(i)$ and may be represented as

$$
\begin{aligned}
e_{k c m}(i) & =e_{k c}(i)-m_{c}\left(\theta_{k}(i)\right) \\
& =\operatorname{Re}\left[\left(z_{k R}(i)-z_{k L}(i)\right) \hat{d}_{k}^{*} e^{-j \hat{\phi}_{k}}\right]-m_{c}\left(\theta_{k}(i)\right)
\end{aligned}
$$

where the subscript $m$ denotes a modification. In (10), the actual DOA, $\theta_{k}(i)$ is needed to estimate a modification factor. However, $\theta_{k}(i)$ is not available in the DOA tracking scheme. In the DiLL scheme, the DOA tracking value $\hat{\theta}_{k}(i)$ is used in generating the modification factor estimation. Thus, (10) may be changed to

$$
\begin{aligned}
\hat{e}_{k c m}(i) & =\operatorname{Re}\left[\left(z_{k R}(i)-z_{k L}(i)\right) d_{k}^{*} e^{-j \phi_{k}}\right]-m_{c}\left(\hat{\theta}_{k}(i)\right) \\
& =G_{c m}\left(\hat{\theta}_{k}(i) \mid \theta_{k}(i)\right)+w_{k c}(i)
\end{aligned}
$$

where $G_{c m}\left(\hat{\theta}_{k}(i) \mid \theta_{k}(i)\right)$ is defined as $G_{c}\left(\hat{\theta}_{k}(i) \mid \theta_{k}(i)\right)$ $m_{c}\left(\hat{\theta}_{k}(i)\right)$, and its characteristic plot is called the modified S-curve and shown for $\theta_{k}(i)$ is $60^{\circ}$ in Fig. 2(b) with the dashed line. Note that it has a zero value when $\hat{\theta}_{k}(i)$ is $60^{\circ}$. The DOA tracking value at the $(i+1)^{\text {th }}$ time may be expressed as

$$
\hat{\theta}_{k}(i+1)=\hat{\theta}_{k}(i)+K_{0} \cdot\left(\hat{e}_{k c m}(i) \otimes f(i)\right) .
$$

\section{Characteristics of the Coherent DiLL}

The coherent DiLL scheme has two spatial correlators as shown in Fig. 1. The modified error signal in (11) may be represented as using (4) and (11)

$$
\begin{aligned}
& \hat{e}_{k c m}(i) \\
= & \operatorname{Re}\left[\frac{\hat{d}_{k}^{*}(i) e^{-j \hat{\phi}_{k}}}{N}\left(\mathbf{a}\left(\hat{\theta}_{k}(i)+\Delta \theta\right)-\mathbf{a}\left(\hat{\theta}_{k}(i)-\Delta \theta\right)\right)^{H} \mathbf{r}_{k}(i)\right] \\
& -\operatorname{Re}\left[\frac{1}{N}\left(\mathbf{a}\left(\hat{\theta}_{k}(i)+\Delta \theta\right)-\mathbf{a}\left(\hat{\theta}_{k}(i)-\Delta \theta\right)\right) \mathbf{a}\left(\hat{\theta}_{k}(i)\right)\right] . \quad \text { (13) }
\end{aligned}
$$

Therefore, one spatial correlator with $\mathbf{a}\left(\hat{\theta}_{k}(i)+\Delta \theta\right)-\mathbf{a}$ $\left(\hat{\theta}_{k}(i)-\Delta \theta\right)$ may replace two spatial correlators with

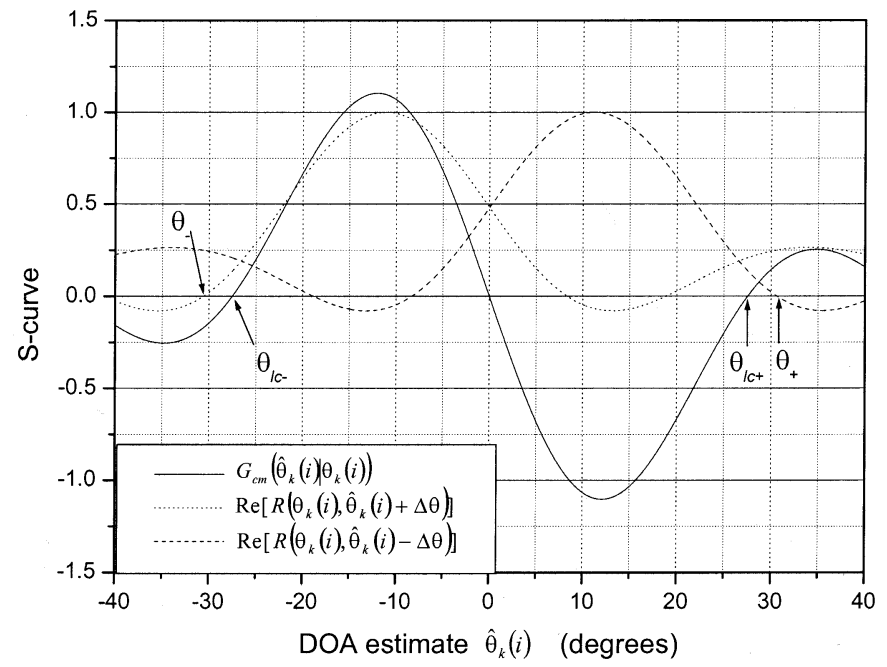

Fig. 3. Locking range.

$\mathbf{a}\left(\hat{\theta}_{k}(i)+\Delta \theta\right)$ and $\mathbf{a}\left(\hat{\theta}_{k}(i)-\Delta \theta\right)$ to reduce the computational requirements.

Note that the DOA tracking value $\hat{\theta}_{k}(i)$ converges to an actual DOA, $\theta_{k}(i)$, when the initial DOA tracking value is in a certain range which is called the locking range. Fig. 3 shows the modified S-curve, $\operatorname{Re}\left[R\left(\theta_{k}(i), \theta_{k}(i)+\Delta \theta\right)\right]$, and $\operatorname{Re}\left[R\left(\theta_{k}(i), \theta_{k}(i)-\Delta \theta\right)\right]$, when $\theta_{k}(i)$ is, $0^{\circ}, N$ is 4 and $\Delta \theta$ is 11.14 ${ }^{\circ}$. As shown in Fig. $3, \theta_{l c+}$ is the smallest positive value of $\hat{\theta}_{k}(i)$, at which the modified S-curve is zero. Similarly, $\theta_{l c-}$ is the largest negative value of $\hat{\theta}_{k}(i)$, at which the modified $\mathrm{S}$-curve is zero. In this paper, the locking range for the coherent DiLL is defined as $\left(\theta_{l c-}, \theta_{l c+}\right)$. However, it is difficult to exactly determine $\theta_{l c+}$ and $\theta_{l c-}$. One solution is to approximate $\theta_{l c+}$ as $\theta_{+}$, which is the smallest positive value of $\hat{\theta}_{k}(i)$, at which $\operatorname{Re}\left[R\left(\theta_{k}(i), \theta_{k}(i)-\Delta \theta\right)\right]$ is zero. Similarly, $\theta_{l c-}$ may be approximated as $\theta_{-}$, which is the largest negative value of $\hat{\theta}_{k}(i)$, at which $\operatorname{Re}\left[R\left(\theta_{k}(i), \hat{\theta}_{k}(i)+\Delta \theta\right)\right]$ is zero. $\theta_{+}$and $\theta_{-}$ should satisfy the following two equations:

$$
\begin{aligned}
& \pi\left(\sin (\theta)-\sin \left(\theta_{+}-\Delta \theta\right)\right)=-\frac{\pi}{(N-1)} \\
& \pi\left(\sin (\theta)-\sin \left(\theta_{-}+\Delta \theta\right)\right)=\frac{\pi}{(N-1)} .
\end{aligned}
$$

From the definition of the modified S-curve in (11), it is always zero when $\hat{\theta}_{k}(i)$ is $\pm 90^{\circ}$. From these results, $\theta_{l c+}$ and $\theta_{l c-}$ are approximated as

$$
\begin{aligned}
& \theta_{l c+} \approx \min \left[\sin ^{-1}\left(\sin \left(\theta_{k}(i)\right)+\frac{1}{N-1}\right)+\Delta \theta, 90^{\circ}\right] \\
& \theta_{l c-} \approx \max \left[\sin ^{-1}\left(\sin \left(\theta_{k}(i)\right)-\frac{1}{N-1}\right)-\Delta \theta,-90^{\circ}\right] .
\end{aligned}
$$

$$
\begin{aligned}
G_{c}\left(\theta_{k}(i) \mid \theta_{k}(i)\right)= & \operatorname{Re}\left[R\left(\theta_{k}(i), \theta_{k}(i)+\Delta \theta\right)-R\left(\theta_{k}(i), \theta_{k}(i)-\Delta \theta\right)\right] \\
=\frac{2}{N} \sum_{n=1}^{N-1}\{ & \sin \left(\frac{1}{2} \pi n\left(\left(\sin \left(\theta_{k}(i)\right)-\sin \left(\theta_{k}(i)-\Delta \theta\right)\right)-\left(\sin \left(\theta_{k}(i)+\Delta \theta\right)-\sin \left(\theta_{k}(i)\right)\right)\right)\right) \\
\cdot & \left.\sin \left(\frac{1}{2} \pi n\left(\sin \left(\theta_{k}(i)+\Delta \theta\right)-\sin \left(\theta_{k}(i)-\Delta \theta\right)\right)\right)\right\}
\end{aligned}
$$




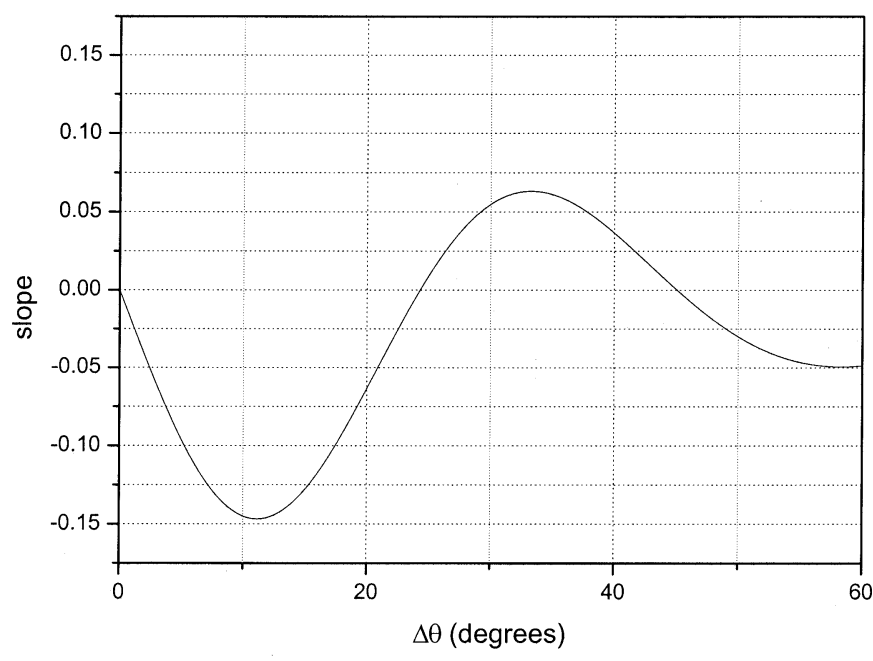

(a)

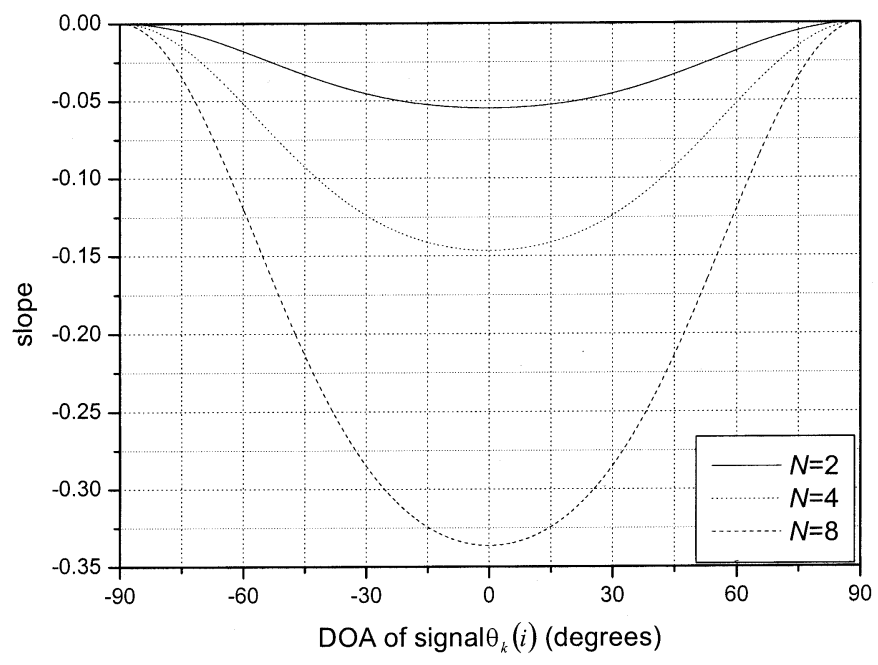

(b)

Fig. 4. Slope of the modified coherent S-curve. (a) Slope of the modified S-curve versus $\Delta \theta\left(\theta=0^{\circ}, N=4\right)$. (b) Slope of the modified S-curve versus $\theta_{k}(i)$

From (15), it is shown that the locking range is inversely proportional to $N$, and increases as $\Delta \theta$ increases. This approximation becomes more accurate as $N$ increases.

The slope of the modified S-curve when $\hat{\theta}_{k}(i)$ is $\theta_{k}(i)$ plays an important role in the DiLL scheme like the DLL scheme [15]-[17]. The slope of the coherent modified S-curve, $s_{c m}\left(\theta_{k}(i)\right)$ at $\hat{\theta}_{k}(i)=\theta_{k}(i)$ is calculated as $s_{c m}\left(\theta_{k}(i)\right)=\left.\left(\partial G_{c m}\left(\hat{\theta}_{k}(i) \mid \theta_{k}(i)\right) / \partial \hat{\theta}_{k}(i)\right)\right|_{\hat{\theta}_{k}(i)=\theta_{k}(i)}$. Fig. 4(a) shows the slope of the modified S-curve as a function of $\Delta \theta$, when $\theta_{k}(i)$ is $0^{\circ}$ and $N$ is 4 . The slope value oscillates between positive and negative values. For the DiLL scheme to operate properly, $\Delta \theta$ should be chosen accordingly to make the slope value negative because the DOA tracking values diverge from the DOA of signals when the slope value is positive. $\Delta \theta$ values in Table I maximize the magnitude of $s_{\mathrm{cm}}\left(\theta_{k}(i)\right)$, when $\theta_{k}(i)$ is $0^{\circ}$ for $N=2,4$ and 8 . Fig. 4(b) shows the slope of the modified S-curve as a function of $\theta_{k}(i)$ for $N=2,4$, and 8 with $\Delta \theta$ values in Table I. As shown in Fig. 4(b), $s_{c m}\left(\theta_{k}(i)\right)$ are negative values for any $\theta_{k}(i)^{\prime}$ 's in $\left(-90^{\circ}, 90^{\circ}\right)$.
TABLE I

$\Delta \theta$

\begin{tabular}{l|c|c}
\hline \multirow{2}{*}{} & \multicolumn{2}{|c}{$\Delta \theta$ (degrees) } \\
\cline { 2 - 3 } & Coherent DiLL & Noncoherent DiLL \\
\hline $\mathrm{N}=2$ & $30.01^{\circ}$ & $30.01^{\circ}$ \\
\hline $\mathrm{N}=4$ & $11.14^{\circ}$ & $12.47^{\circ}$ \\
\hline $\mathrm{N}=8$ & $5.09^{\circ}$ & $6.01^{\circ}$ \\
\hline
\end{tabular}

\section{Computational Requirements}

The coherent DiLL scheme requires two vector inner product operations for the calculations of $e_{k c}(i)$ and $m_{c}\left(\hat{\theta}_{k}(i)\right)$ for each signal source as shown in (13). Thus, the coherent DiLL scheme requires $2 N K+\mathrm{O}(K)$ operations every update, where $N$ is the number of antenna elements and $K$ is the number of signal sources. To reduce computational requirement, $m_{c}\left(\hat{\theta}_{k}(i)\right)$ can be stored as a function of $\hat{\theta}_{k}(i)$ in a table. Therefore, the required complex operation for the coherent DiLL is reduced to $N K+\mathrm{O}(K)$ which is less than that of the PASTd described in [5].

\section{NONCOHERENT DiLL}

In this section, we present the noncoherent DiLL, and investigate the characteristics of the noncoherent DiLL in comparison with that of the coherent DiLL.

\section{A. Principles of the Noncoherent DiLL}

In the noncoherent DiLL scheme, an error signal needed in updating the DOA tracking value may be generated from the squared correlator outputs instead of the real part of the correlator outputs as in the coherent mode [20]. The noncoherent DiLL does not need carrier recovery or transmitted data estimation.

The despread signal vector $\mathbf{r}_{k}(i)$ is spatially correlated simultaneously with right-shifted and left-shifted array response vectors a $\left(\hat{\theta}_{k}(i)+\Delta \theta\right)$ and $\mathbf{a}\left(\hat{\theta}_{k}(i)-\Delta \theta\right)$. The difference between the squared correlator outputs generates the error signal $e_{k n}(i)$ which is defined as

$e_{k n}(i)=\left|z_{k R}(i)\right|^{2}-\left|z_{k L}(i)\right|^{2}=G_{n}\left(\hat{\theta}_{k}(i) \mid \theta_{k}(i)\right)+w_{k n}(i)$

where $\quad G_{n}\left(\hat{\theta}_{k}(i) \mid \theta_{k}(i)\right) \quad$ is defined as $\left|R\left(\theta_{k}(i), \hat{\theta}_{k}(i)+\Delta \theta\right)\right|^{2}-\left|R\left(\theta_{k}(i), \hat{\theta}_{k}(i)-\Delta \theta\right)\right|^{2}$, its plot is referred to as the noncoherent S-curve, the noise and MAI terms $w_{k n}(i)$ may be defined as

$$
\begin{aligned}
w_{k n}(i)= & \left|I_{k R}(i)\right|^{2}-\left|I_{k L}(i)\right|^{2}+\left|v_{k R}(i)\right|^{2}-\left|v_{k L}(i)\right|^{2} \\
& +2 \operatorname{Re}\left[d_{k}^{*}(i) R^{*}\left(\theta_{k}(i), \hat{\theta}_{k}(i)+\Delta \theta\right)\right. \\
& \left.\cdot\left(I_{k R}(i)+v_{k R}(i)\right)+I_{k R}^{*}(i) v_{k R}(i)\right] \\
& -2 \operatorname{Re}\left[d_{k}^{*}(i) R^{*}\left(\theta_{k}(i), \hat{\theta}_{k}(i)-\Delta \theta\right)\right. \\
& \left.\cdot\left(I_{k L}(i)+v_{k L}(i)\right)+I_{k L}^{*}(i) v_{k L}(i)\right] \\
& z_{k R}(i), z_{k L}(i), I_{k R}(i), I_{k L}(i), v_{k R}(i)
\end{aligned}
$$

and $v_{k R}(i)$ are defined in (4), and the subscript $n$ denotes a noncoherent mode. $w_{k n}(i)$ is composed of the MAI terms, noise 
terms, and the cross-product terms among the desired source's signal, MAI, and noise terms.

As explained in the coherent DiLL, the error signal should be modified to remove the bias by subtracting a modification factor as follows:

$$
\begin{aligned}
\hat{e}_{k n m}(i) & =G_{n}\left(\hat{\theta}_{k}(i) \mid \theta_{k}(i)\right)-m_{n}\left(\hat{\theta}_{k}(i)\right)+w_{k n}(i) \\
& =G_{n m}\left(\hat{\theta}_{k}(i) \mid \theta_{k}(i)\right)+w_{k n}(i)
\end{aligned}
$$

where the noncoherent modification factor, $m_{n}\left(\hat{\theta}_{k}(i)\right)$ is defined as $G_{n}\left(\hat{\theta}_{k}(i) \mid \hat{\theta}_{k}(i)\right)$ and the modified noncoherent S-curve is represented as $G_{n}\left(\hat{\theta}_{k}(i) \mid \theta_{k}(i)\right)-m_{n}\left(\hat{\theta}_{k}(i)\right)$, respectively. The modified error signal is filtered and fed to the numerically controlled oscillator (NCO) to update the DOA tracking value iteratively. The DOA tracking value at the $(i+1)^{\text {th }}$ time is updated using the following adaptive equation:

$$
\hat{\theta}_{k}(i+1)=\hat{\theta}_{k}(i)+K_{0} \cdot\left(\hat{e}_{k n m}(i) \otimes f(i)\right)
$$

where $\mathrm{K}_{\mathrm{o}}$ is the $\mathrm{NCO}$ gain, $e_{k n m}(i)$ is the modified noncoherent error signal, and $f(i)$ is the impulse response of the loop filter. The iterative updating of a DOA tracking value also enables the noncoherent DiLL to track the DOA of a moving source.

\section{B. Characteristics of the Noncoherent DiLL}

The locking range of the noncoherent DiLL is also approximated as $\left(\theta_{\ln -}, \theta_{\ln +}\right)$, and is defined as the range between the two zero crossing points of $G_{n}\left(\hat{\theta}_{k}(i) \mid \theta_{k}(i)\right)$, which are closest to $\hat{\theta}_{k}(i)=\theta_{k}(i)$ on the left and right sides. Fig. 5(a) shows $G_{n}\left(\hat{\theta}_{k}(i) \mid \theta_{k}(i)\right)$ as a function of $\hat{\theta}_{k}(i)$, when $\hat{\theta}_{k}(i)$ is $0^{\circ}, N$ is 4 , and $\Delta \theta$ is $12.47^{\circ}$. We may express the approximated values of $\theta_{\ln }+$ and $\theta_{\ln -}$, as follows [19]:

$$
\begin{aligned}
& \theta_{\mathrm{ln}+} \approx \min \left[\sin ^{-1}\left(\sin \left(\theta_{k}(i)\right)+\frac{2}{N}\right)+\Delta \theta, 90^{\circ}\right] \\
& \theta_{\mathrm{ln}-} \approx \max \left[\sin ^{-1}\left(\sin \left(\theta_{k}(i)\right)-\frac{2}{N}\right)-\Delta \theta,-90^{\circ}\right] .
\end{aligned}
$$

Comparing (19) with (15), it is found that the locking range of the noncoherent DiLL for $N \geq 2$ is equal to or wider than that of the coherent DiLL, because the real part has a smaller azimuth range than that of the squared [18].

As explained in previous section, the negative slope condition of $G_{n m}\left(\hat{\theta}_{k}(i) \mid \theta_{k}(i)\right)$ is required to ensure that $\hat{\theta}_{k}(i)$ converges to $\theta_{k}(i)$. The slope of $G_{n m}\left(\hat{\theta}_{k}(i) \mid \theta_{k}(i)\right), \quad s_{n m}\left(\theta_{k}(i)\right), \quad$ is defined as $\left.s_{n m}\left(\theta_{k}(i)\right) \triangleq\left(\partial G_{n m}\left(\hat{\theta}_{k}(i) \mid \theta_{k}(i)\right) / \partial \hat{\theta}_{k}(i)\right)\right|_{\hat{\theta}_{k}(i)=\theta_{k}(i)}$.

Fig. 5(b) shows the plot of $s_{n m}\left(\theta_{k}(i)\right)$ as a function of $\theta_{k}(i)$, when $N$ is 2,4 , and 8 with $\Delta \theta$ values in Table I which maximize the magnitude of the modified noncoherent $\mathrm{S}$-curve slope. The negative slope value of $G_{n m}\left(\hat{\theta}_{k}(i) \mid \theta_{k}(i)\right)$ ensures that the DOA tracking value converges to an actual DOA $\theta_{k}(i)$, when the initial DOA tracking value is in a locking range.

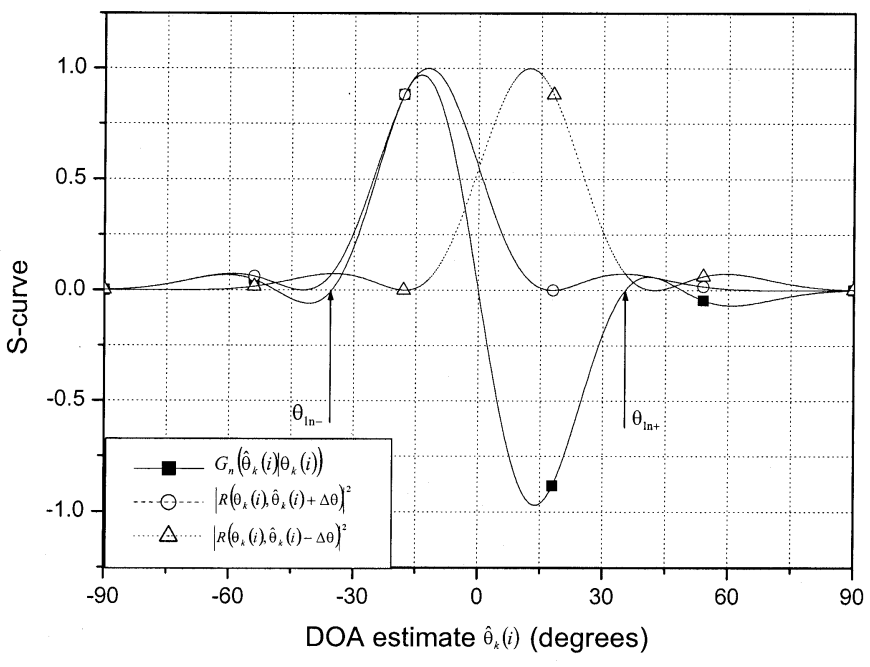

(a)

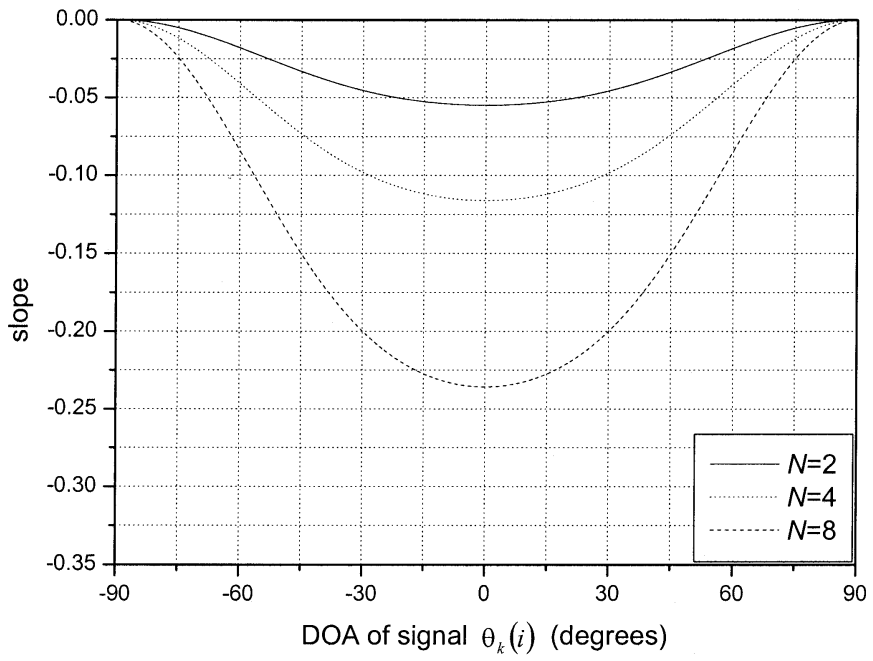

(b)

Fig. 5. Characteristics of the modified noncoherent S-curve. (a) Modified noncoherent S-curve. (b) Slope of the modified noncoherent S-curve.

Comparing Figs. 4(b) and 5(b), the amplitude of the modified S-curve slope of the noncoherent DiLL scheme is less than that of the coherent DiLL scheme. From these results, the tracking accuracy of the coherent scheme is better than the noncoherent DiLL scheme which will be explained in more detail. However, the data and carrier phase estimation are not required for the noncoherent scheme.

\section{Computational Requirements}

The noncoherent DiLL scheme requires four vector inner product operations for the calculations of $z_{k R}(i), z_{k L}(i)$, and $m_{n}\left(\hat{\theta}_{k}(i)\right)$ for each signal source as shown in (17). Thus, the noncoherent DiLL scheme requires $4 N K+\mathrm{O}(K)$ operations for each update. To reduce computational requirement, $m_{n}\left(\hat{\theta}_{k}(i)\right)$ can be stored as a function of $\hat{\theta}_{k}(i)$ in a table as explained in the coherent DiLL. Therefore, the required operation for the noncoherent DiLL is reduced to $2 N K+\mathrm{O}(K)$ which is also less than that of the PASTd but has a higher computational requirement that of the coherent DiLL. 


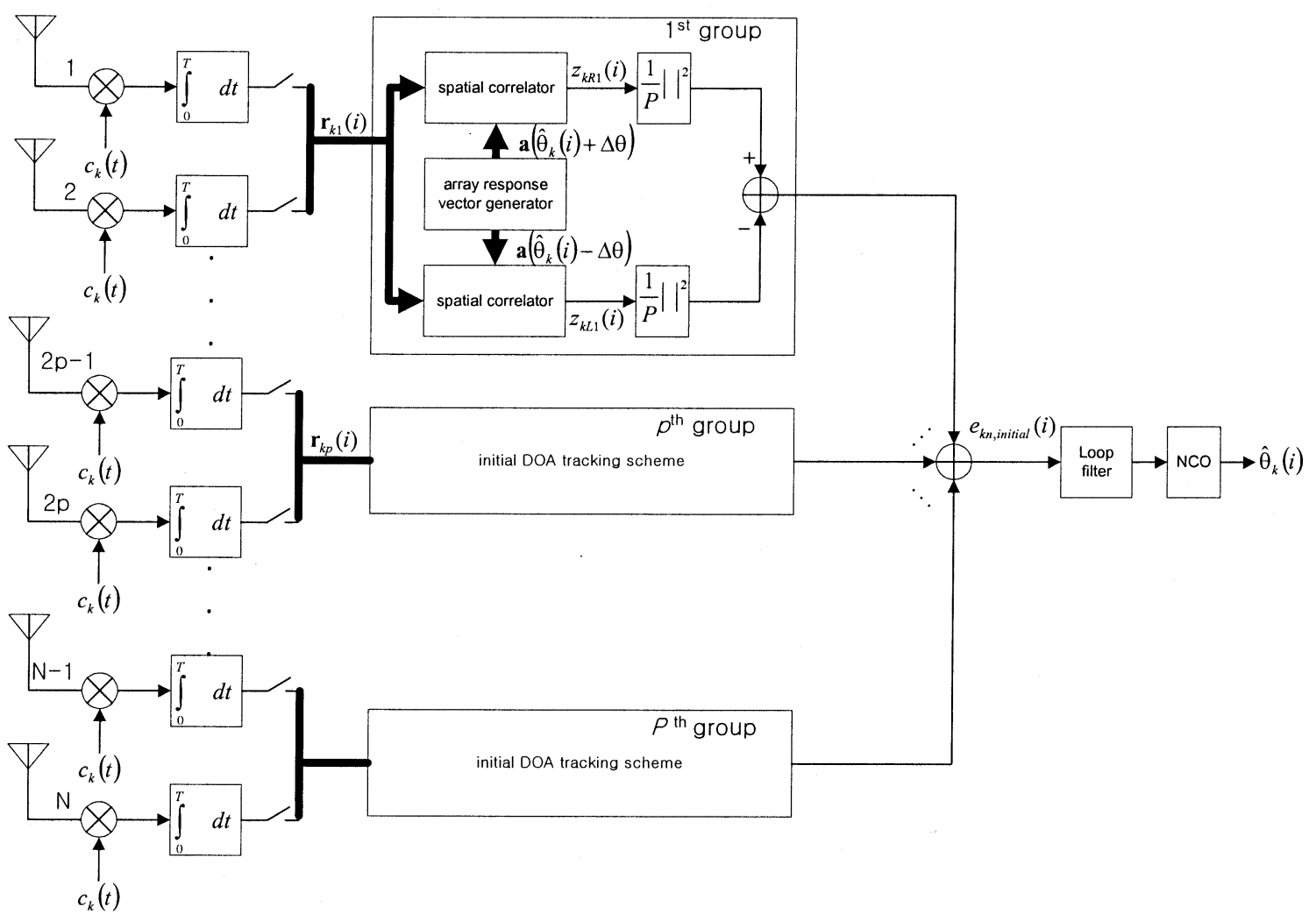

(a)

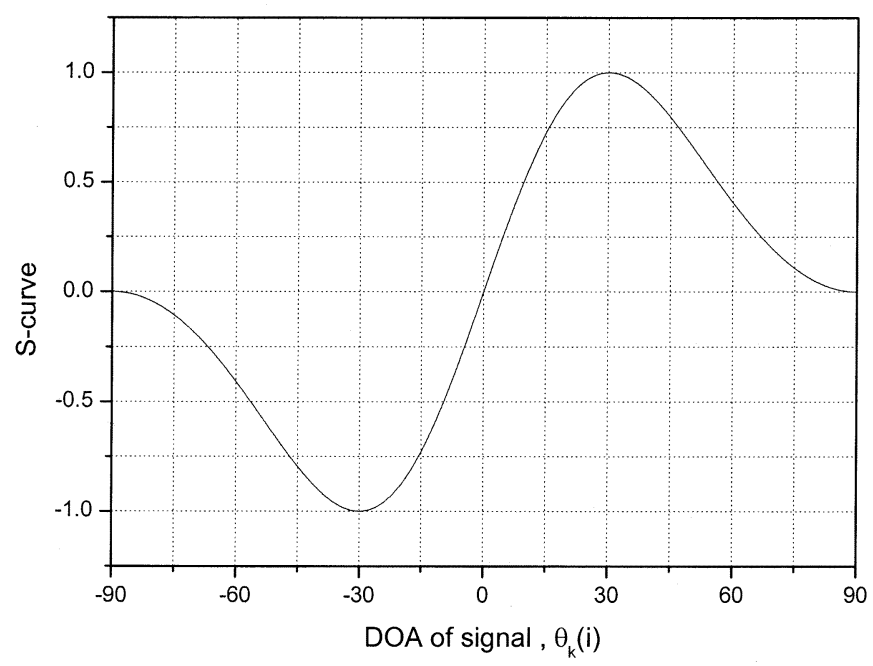

(b)

Fig. 6. Initial DOA tracking scheme. (a) Block diagram of the initial DOA tracking scheme. (b) Noncoherent $\mathrm{S}$-curve value $\left(N=2, \Delta \theta=30.01^{\circ}, \hat{\theta}_{k}(i)=\right.$ $0^{\circ}$ ).

\section{INITIAL DOA TRACKING}

As mentioned before, in order to converge to an actual DOA $\theta_{k}(i)$, the initial DOA tracking value should be in the locking range. Assuming that the DOA of a signal is within the range from $-90^{\circ}$ to $90^{\circ}$, we propose an initial DOA tracking method by modifying the DiLL scheme in this section.

Note that in the case of two antenna elements the lower bounds of the locking range, $\theta_{l c-}$ and $\theta_{\mathrm{ln}}$ - are less than $0^{\circ}$ when $\theta_{k}(i)$ is larger than $0^{\circ}$. In contrast, when $\theta_{k}(i)$ is less than $0^{\circ}$, the upper bounds of the locking range, $\theta_{l c+}$ and $\theta_{\ln }+$ are larger than $0^{\circ}$. Hence, the locking range for the signal with DOA from $-90^{\circ}$ to $90^{\circ}$ includes $0^{\circ}$ in both DiLL schemes with two antenna elements, and the DOA tracking value converges to the DOA of a signal by using the DiLL scheme with the initial DOA tracking value of $0^{\circ}$. When there are $N$ antenna elements, $N$ elements may be divided into groups of two elements, as shown in Fig. 6(a). The error signals generated by each group are then combined. 
Let $\mathbf{r}_{k p}(i)$ denote the vector of the received signal at the $p^{\text {th }}$ group for the $k^{\text {th }}$ user signal vector. Following the notation of (3), we may write

$$
\begin{aligned}
\mathbf{r}_{k p}(i)= & e^{-j 2(p-1) \pi \sin \left(\theta_{k}(i)\right)} d_{k}(i) \mathbf{a}_{2}\left(\theta_{k}(i)\right) e^{j \phi_{k}} \\
& +\sum_{\substack{l=1 \\
l \neq k}}^{K} e^{-j 2(p-1) \pi \sin \left(\theta_{l}(i)\right)} \psi_{k l} d_{l}(i) \mathbf{a}_{2}\left(\theta_{l}(t)\right) e^{j \phi_{l}} \\
& +\mathbf{v}_{k p}(i)
\end{aligned}
$$

where $\mathbf{a}_{2}\left(\theta_{k}(i)\right)=\left[1, e^{-j \pi \sin \left(\theta_{k}(i)\right)}\right]^{\mathrm{T}}$ and $\mathbf{v}_{k p}(i)=$ $\left[\begin{array}{ll}v_{k, 2 p-1}(i) & v_{k, 2 p}(i)\end{array}\right]^{\mathrm{T}}$. Each group's received signal vector is spatially correlated with $\mathbf{a}_{2}\left(\hat{\theta}_{k}(i)+\Delta \theta\right)$ and $\mathbf{a}_{2}\left(\hat{\theta}_{k}(i)-\Delta \theta\right)$. The spatial correlator output may be represented as

$$
\begin{aligned}
& z_{k R p}(i) \\
= & e^{-j 2(p-1) \pi \sin \left(\theta_{k}(i)\right)+j \phi_{k}} d_{k}(i) R_{2}\left(\theta_{k}(i), \hat{\theta}_{k}(i)+\Delta \theta\right) \\
& +\sum_{\substack{l=1 \\
l \neq k}}^{K} e^{-j 2(p-1) \pi \sin \left(\theta_{l}(i)\right)+j \phi_{l}} \psi_{k l} d_{l}(i) R_{2}\left(\theta_{l}(i) \hat{\theta}_{k}(i)+\Delta \theta\right) \\
& +v_{k R p}(i), \\
& z_{k L p}(i) \\
= & e^{-j 2(p-1) \pi \sin \left(\theta_{k}(i)\right)+j \phi_{k}} d_{k}(i) R_{2}\left(\theta_{k}(i), \hat{\theta}_{k}(i)-\Delta \theta\right) \\
& +\sum_{\substack{l=1 \\
l \neq k}}^{K} e^{-j 2(p-1) \pi \sin \left(\theta_{l}(i)\right)+j \phi_{l}} \psi_{k l} d_{l}(i) R_{2}\left(\theta_{l}(i) \hat{\theta}_{k}(i)-\Delta \theta\right) \\
& +v_{k L p}(i)
\end{aligned}
$$

where $R_{2}\left(\theta_{1}, \theta_{2}\right)$ is defined as $(1 / 2) \mathbf{a}_{2}^{H}\left(\theta_{2}\right) \mathbf{a}_{2}\left(\theta_{1}\right)$, $v_{k R p}(i)=(1 / 2) \mathbf{a}_{2}^{H}\left(\hat{\theta}_{k}(i)+\Delta \theta\right) \mathbf{v}_{k p}(i)$ and $v_{k L p}(i)=$ $(1 / 2) \mathbf{a}_{2}^{H}\left(\hat{\theta}_{k}(i)-\Delta \theta\right) \mathbf{v}_{k p}(i)$. Due to the phase difference between each group, $e^{-j 2(p-1) \pi \sin \left(\theta_{k}(i)\right)+j \phi_{k}}$, the noncoherent combining method is used in the initial DOA tracking scheme. Thus, the combined error signal may be represented as

$e_{k n, \text { initial }}(i)=\frac{1}{P} \sum_{p=1}^{P}\left\{\left|z_{k R p}(i)\right|^{2}-\left|z_{k L p}(i)\right|^{2}\right\}-m_{n 2}\left(\hat{\theta}_{k}(i)\right)$

where $P$ is the number of groups and $m_{n 2}\left(\hat{\theta}_{k}(i)\right)$ is the noncoherent modification factor with two antenna elements which is defined as $\left|R_{2}\left(\hat{\theta}_{k}(i), \hat{\theta}_{k}(i)+\Delta \theta\right)\right|^{2}-$ $\left|R_{2}\left(\hat{\theta}_{k}(i), \hat{\theta}_{k}(i)-\Delta \theta\right)\right|^{2}$. This combined error signal passes the loop filter and is fed to the NCO to update the DOA tracking value.

After the initial DOA tracking mode, an $N$-antenna-elements-based DiLL scheme will be used for the DOA tracking to enhance the tracking accuracy. The tracking accuracy is approximately proportional to the number of antenna elements, which will be explained in the next section.

Fig. 6(b) shows the modified S-curve value at $\hat{\theta}_{k}(i)=0^{\circ}$ as a function of $\theta_{k}(i)$. Note that when the DOA of a signal is near $-90^{\circ}$ or $+90^{\circ}$, the error signal is very small, thus, the error signal is vulnerable to noise and MAI terms. In this paper, the
DOA of signals is restricted between $\left(-60^{\circ}, 60^{\circ}\right)$, assuming sectorization on the antenna elements which is typical of a common cell sectorization scheme.

\section{Performance Analysis and Numerical Results}

\section{A. Mean Square Error (MSE) of the Coherent DiLL Scheme}

In this section, we analyze the MSE of the coherent DiLL scheme for the stationary sources in the steady state. Generally, this steady-state analysis provides the lower bound of the MSE of tracking scheme because the MSE of stationary source is less than that of the moving source.

The procedure for analyzing the MSE of DOA tracking in the case of the coherent DiLL scheme is similar to that of the coherent DLL [17]. The DOA tracking error for the $k$ th user at the $i$ th time is defined as $\varepsilon_{k}(i)=\theta_{k}-\hat{\theta}_{k}(i)$ where $\theta_{k}$ is the DOA of the stationary source. The update formula (12) may be rewritten as

$\varepsilon_{k}(i+1)=\varepsilon_{k}(i)-K_{0} \cdot\left(G_{c m}\left(\hat{\theta}_{k}(i) \mid \theta_{k}\right)+w_{k c}(i)\right) \otimes f(i)$.

With the assumption that the DOA tracking error is small, $G_{c m}\left(\hat{\theta}_{k}(i) \mid \theta_{k}\right)$ may be approximated as a linear function of $\varepsilon_{k}(i)$ such that $G_{c m}\left(\hat{\theta}_{k}(i) \mid \theta_{k}\right) \approx-s_{c m}\left(\theta_{k}\right) \varepsilon_{k}(i)$, and $\varepsilon_{k}(i+1)$ may be written as

$$
\begin{aligned}
\varepsilon_{k}(i+1)=\varepsilon_{k}(i)+ & K_{0} s_{c m}\left(\theta_{k}\right) \\
\cdot & {\left[\left(\varepsilon_{k}(i)+\frac{w_{k c}(i)}{s_{c m}\left(\theta_{k}\right)}\right) \otimes f(i)\right] . }
\end{aligned}
$$

The DOA tracking error may be written in the $z$-domain form as

$$
\mathrm{E}_{k}(z)=\frac{-K_{0} s_{c m}\left(\theta_{k}\right) F(z) z^{-1}}{1-\left(1+K_{0} s_{c m}\left(\theta_{k}\right) F(z)\right) z^{-1}} \cdot\left(\frac{W_{k c}(z)}{s_{c m}\left(\theta_{k}\right)}\right) .
$$

As a result, the MSE of DOA tracking in the steady state, $\sigma_{\varepsilon_{s}}^{2}$ is represented as

$$
\sigma_{\varepsilon_{s}}^{2}=\frac{\operatorname{Var}\left(w_{k c}(i)\right) B_{L c}}{s_{c m}^{2}\left(\theta_{k}\right)}=\frac{B_{L c}}{s_{c m}^{2}\left(\theta_{k}\right)} \cdot \frac{\xi_{c}}{\rho N}
$$

where $\xi_{k c}=\left(1-\operatorname{Re}\left(R\left(\theta_{k}+\Delta \theta, \theta_{k}-\Delta \theta\right)\right)\right)$ and $B_{L c}$ is two-sided noise bandwidth of the closed-loop transfer function,

$$
H(z) \triangleq \frac{-K_{0} s_{c m}\left(\theta_{k}\right) F(z) z^{-1}}{1-\left(1+K_{0} s_{c m}\left(\theta_{k}\right) F(z)\right) z^{-1}}
$$

in (24). Note that it is found that the variance of $w_{k c}(i)$ in (12) is $\xi_{c} / \rho N$ where $\rho$ is defined as $\rho \triangleq l_{\text {limits }}=1 /\left(\sigma^{2}+(K-1) / L\right)$. In this definition of $\rho$, we assume that the random sequences with length $L$ are used for the spreading sequences, the interfering signal sources have the same power with the desired signal source, and the noise and MAI terms are additive white Gaussian. Since the signal power is assumed to be 1 and the noise variance is $\sigma^{2}$ for each antenna element, as described in Section III, $\rho$ represents SINR in each antenna. Therefore, the MSE of DOA tracking in coherent mode is inversely proportional to SINR and the number antenna elements, $N$. From these results, it is observed that the MSE of DOA tracking becomes larger as the number of signal sources increases or the 
processing gain decreases. In this analysis, we assume that the data and carrier phase estimation is perfect. As the data and carrier phase estimation error increase, the MSE of DOA tracking increases because the signal power is reduced as shown in Section VI-E.

\section{B. MSE of the Noncoherent DiLL Scheme}

In this section, the MSE of DOA tracking for the noncoherent DiLL scheme is analyzed. The DOA tracking error may be represented in the $z$-domain for the stationary source as

$$
\mathrm{E}_{k n}(z)=\frac{-K_{0} s_{n m}\left(\theta_{k}\right) F(z) z^{-1}}{1-\left(1+K_{0} s_{n m}\left(\theta_{k}\right) F(z)\right) z^{-1}} \cdot\left(\frac{W_{k n}(z)}{s_{n m}\left(\theta_{k}\right)}\right) .
$$

The MSE of DOA tracking is represented as

$$
\sigma_{\varepsilon_{s}}^{2}=\frac{\operatorname{Var}\left(w_{k n}(i)\right) B_{L n}}{s_{n m}^{2}\left(\theta_{k}\right)}
$$

where $B_{L n}$ is the two-sided noise bandwidth of the closed-loop transfer function,

$$
H(z) \triangleq \frac{-K_{0} s_{n m}\left(\theta_{k}\right) F(z) z^{-1}}{1-\left(1-K_{0} s_{n m}\left(\theta_{k}\right) F(z)\right) z^{-1}}
$$

in (26). By using (16), the variance of $w_{k n}(i)$ may be approximated as

$$
\begin{array}{r}
\operatorname{Var}\left(w_{k n}(i)\right)=\frac{2}{\rho N} \cdot\left(q\left(\theta_{k}, \varepsilon_{s}\right)-2 \operatorname{Re}\left(R\left(\theta_{k}, \theta_{k}+\Delta \theta\right)\right.\right. \\
\left.\left.\cdot R^{*}\left(\theta_{k}, \theta_{k}-\Delta \theta\right) R\left(\theta_{k}+\Delta \theta, \theta_{k}-\Delta \theta\right)\right)\right)
\end{array}
$$

where $q\left(\theta_{k}, \varepsilon_{s}\right)$ is defined as

$$
\begin{aligned}
q\left(\theta_{k}, \varepsilon_{s}\right) \triangleq E\left[\left|R\left(\theta_{k}, \theta_{k}-\varepsilon_{s}+\Delta \theta\right)\right|^{2}\right. & \\
& \left.+\left|R\left(\theta_{k}, \theta_{k}-\varepsilon_{s}-\Delta \theta\right)\right|^{2}\right]
\end{aligned}
$$

and $\varepsilon_{s}$ denotes the tracking error in the steady state. When $\alpha_{k n}$ and $\gamma_{k n}$ are defined as $\alpha_{k n}=\left.(1 / 2 !) \cdot\left(\partial^{2} q\left(\varepsilon_{s}\right) / \partial \varepsilon_{s}^{2}\right)\right|_{\varepsilon_{s}=0}$ and $\gamma_{k n}=\left.q\left(\theta_{k}, \varepsilon_{s}\right)\right|_{\varepsilon_{s}=0}, q\left(\theta_{k}, \varepsilon_{s}\right)$ may be approximated as $q\left(\theta_{k}, \varepsilon_{s}\right)=\alpha_{k n} \sigma_{\varepsilon_{s}}^{2}+\gamma_{k n}$ [16], [19]. Using (27) and (28), $\sigma_{\varepsilon_{s}}^{2}$ may be represented as (29), shown at the bottom of the page.If we solve for $\sigma_{\varepsilon_{s}}^{2}, \sigma_{\varepsilon_{s}}^{2}$ is further simplified to

$$
\sigma_{\varepsilon_{s}}^{2}=\frac{2 B_{L n}}{s_{n m}^{2}(\theta) \cdot \rho N} \cdot \frac{\xi_{k n}}{\left(1-\frac{2 \alpha_{k n} \cdot B_{L n}}{s_{n m}^{2}\left(\theta_{k}\right) \cdot \rho N}\right)} \cong \frac{2 B_{L n} \cdot \xi_{k n}}{s_{n m}^{2}\left(\theta_{k}\right) \cdot \rho N}
$$

where

$$
\begin{aligned}
& \xi_{k n} \triangleq \gamma_{k n}-2 \operatorname{Re}\left(R\left(\theta_{k}, \theta_{k}+\Delta \theta\right)\right. \\
&\left.\cdot R^{*}\left(\theta, \theta_{k}-\Delta \theta\right) R\left(\theta_{k}+\Delta \theta, \theta_{k}-\Delta \theta\right)\right) .
\end{aligned}
$$

In calculating (30), we assume that $2 \alpha_{k n} B_{L n} / s_{n m}^{2}\left(\theta_{k}\right) \cdot \rho N$ is much less than 1. This result shows that the MSE of DOA tracking in the noncoherent mode is also inversely proportional to SINR and $N$ as in the coherent mode. Comparing (25) and
(30), the noncoherent scheme has the multiplying factor 2 representing the squaring loss. In addition, $s_{c m}^{2}\left(\theta_{k}\right)$ is larger than $s_{n m}^{2}\left(\theta_{k}\right)$. Therefore, the MSE of the noncoherent mode may be larger than that of the coherent mode by the factor of more than 2.

\section{Environments of the Numerical Results}

We investigate the performance of the coherent and noncoherent DiLL schemes and compare the both DiLL schemes with PASTd. In this paper, we assume that the number of antenna elements, $N$ is 8 , SNR per antenna is $5 \mathrm{~dB}$, the number of signal sources is 3 , a random code is used for spreading sequence with the processing gain, $L=32$, and BPSK modulation and spreading are used. A uniform linear array of omnidirectional antenna elements with half-wavelength spacing is used. We set the parameters used in analysis and simulation to make the MSE of DOA tracking of the noncoherent DiLL scheme and PASTd equal for the stationary source in the steady state when $\theta$ is $0^{\circ}$. In the both DiLL schemes, the NCO gain, $K_{\mathrm{o}}$ is 0.05 , and the one-pole IIR filter with forgetting factor 0.9 is used for the loop filter whose $z$-transform is $F(z)=\left(0.1 /\left(1-0.9 z^{-1}\right)\right) \cdot \Delta \theta$ for the both DiLL schemes are listed in Table I. In the PASTd, the forgetting factor is set to 0.97 and the initial value of the eigenvalue estimate is chosen to be one [5]. And, the DOA tracking scheme based on the PASTd in [6] is used for this paper.

We analyze and simulate the MSE of DOA tracking for 3 users as in (25) and (30) for the both DiLL schemes and compare with PASTd. In addition, in case of the coherent DiLL scheme, data estimation and carrier phase error effects on the MSE are also simulated. Finally, the number of iteration required for the initial tracking stage is investigated.

\section{DOA Tracking for a Moving Source}

In this subsection, the MSE of DOA tracking of the both DiLL schemes and PASTd are investigated in the above mentioned environments. Fig. 7 shows the trajectories and the MSEs of DOA tracking of the both DiLL schemes and PASTd. Fig. 7(a) shows the true trajectory of DOA's over time. The first and second signal sources move from $60^{\circ}$ to $55^{\circ}$ and $-60^{\circ}$ to $-50^{\circ}$ with constant angular velocity, respectively, and the third signal source stays at $-55^{\circ}$. The speed of the first signal source corresponds to $200 \mathrm{~km} / \mathrm{h}$ at the $100 \mathrm{~m}$ from the base station when the data rate is $9.6 \mathrm{~kb} / \mathrm{s}$. Fig. 7(b)-(d) shows the MSE of DOA tracking, obtained by ensemble averaging over 500 independent trials for the both DiLL schemes and PASTd, respectively. An initial DOA tracking value is $0^{\circ}$ which is explained in Section V. And, the initial tracking mode length is 50 symbol times which will be shown in Section VI.F. In the case of the coherent DiLL scheme, we assume that the data and carrier phase estimation is perfect.

From Fig. 7(b) and (c), it is observed that the MSE of the coherent DiLL scheme is less than that of the noncoherent

$$
\sigma_{\varepsilon_{s}}^{2}=\frac{2 B_{L n}}{s_{n m}^{2}\left(\theta_{k}\right)} \cdot \frac{\left(\alpha_{k n} \sigma_{\varepsilon_{s}}^{2}+\gamma_{k n}-2 \operatorname{Re}\left(R\left(\theta_{k}, \theta_{k}+\Delta \theta\right) R^{*}\left(\theta_{k}, \theta_{k}-\Delta \theta\right) R\left(\theta_{k}+\Delta \theta, \theta_{k}-\Delta \theta\right)\right)\right)}{\rho N}
$$




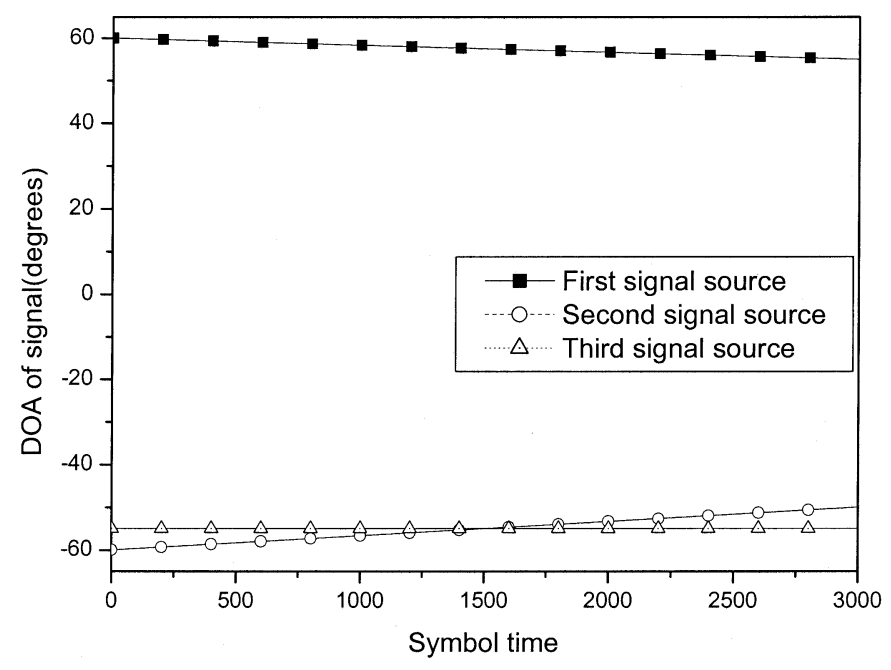

(a)

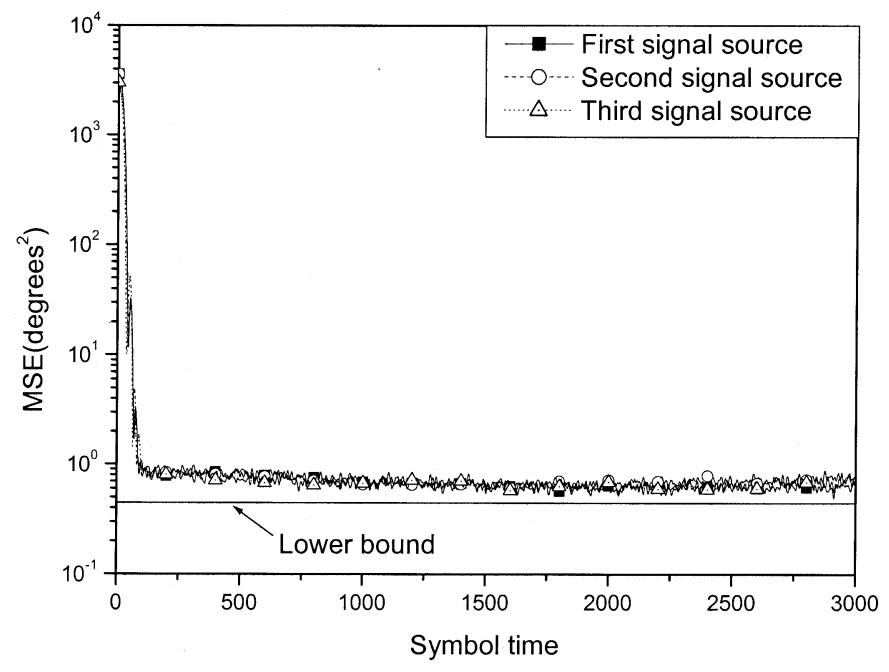

(c)

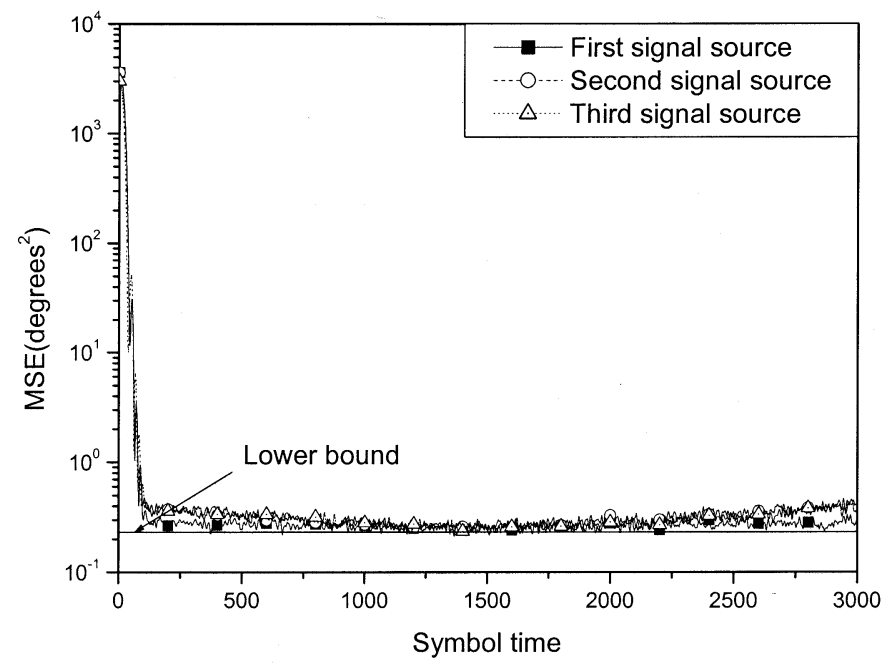

(b)

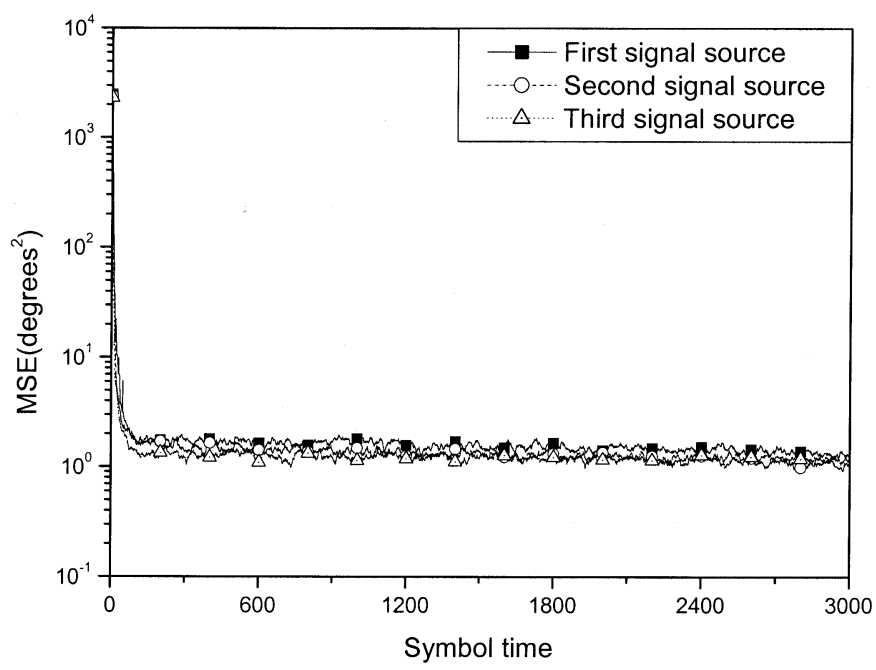

(d)

Fig. 7. IMSE of the DOA tracking error of three users. (a) Trajectories of moving sources. (b) Coherent DiLL scheme. (c) Noncoherent DiLL scheme. 9d) PASTd.

scheme by the factor of more than 2 . This happens because of the squaring loss in the noncoherent mode and the difference of the modified S-curve slope, as explained in the Section VI-B. Note that the analysis results are the lower bounds of the simulation results in the both DiLL schemes. In the both DiLL schemes, the MSE of three signal sources are almost the same regardless of the sources' motion.

From Fig. 7(c) and (d), the noncoherent DiLL schemes have a little less MSE of DOA tracking than those of the PASTd even though the parameters are set to make the MSE of DOA tracking equal. In the initial tracking stage, the DiLL and PASTd show the similar performance. From these results, it is observed that the both DiLL schemes have better tracking capabilities than the PASTd.

\section{E. Effects of Data and Carrier Phase Estimation Error}

The effects of data and carrier phase estimation error on the coherent DiLL scheme are presented in Fig. 8. In the figure, vertical axis denotes the total averaged MSE of DOA tracking for three signal sources with the same environments as in the previous section.

Fig. 8(a) shows the MSE of DOA tracking as a function of data estimation error rate with the assumption that the data estimation error occurs randomly and the carrier phase estimation is perfect in (6). Since the data estimation is not required in the noncoherent mode as in (16), the MSE of the noncoherent mode is not affected by the data estimation error rate. However, the MSE of the coherent DiLL increases as the data estimation error rates increases because the signal powers are reduced by the data estimation error. The coherent DiLL has smaller DOA tracking error variance than the noncoherent DiLL as long as the data estimation error is less than 0.16 as shown in Fig. 8(a).

Fig. 8(b) shows the MSE of DOA tracking as a function of standard deviation of carrier phase error. In this simulation, it is assumed that the carrier phase error, defined as $\phi_{k}-\hat{\phi}_{k}$, is Gaussian distributed random variable and the data estimation is perfect. The MSE of the noncoherent DiLL scheme is not affected by the carrier phase estimation error because of the squaring operation in (16). However, the MSE of the coherent DiLL increases as the carrier phase error increases because the 


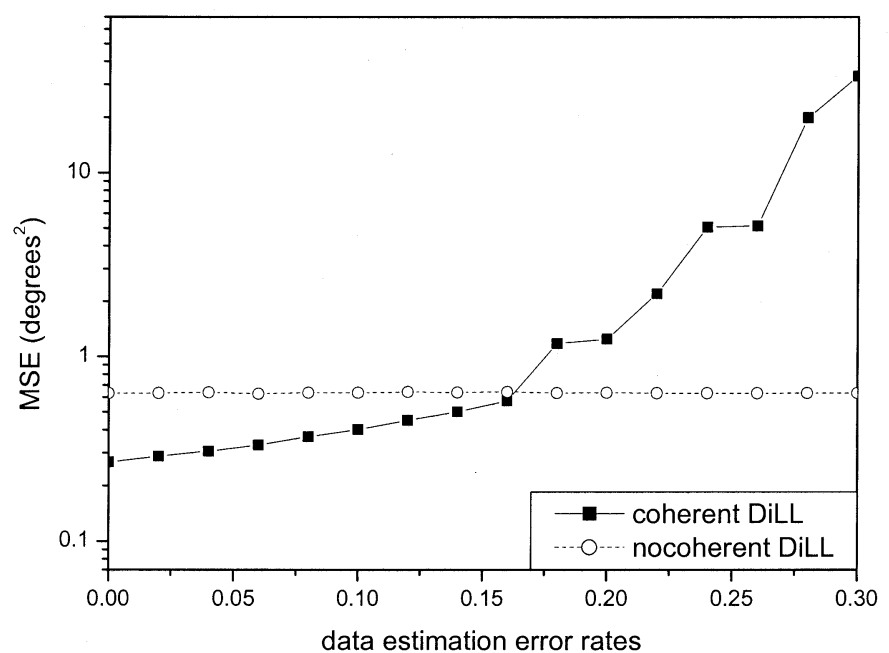

(a)

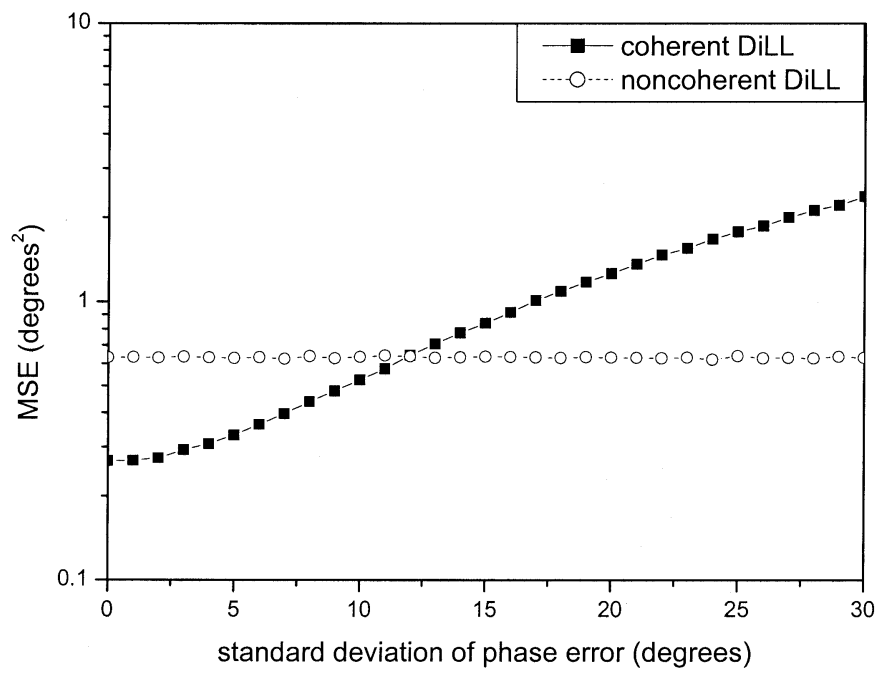

(b)

Fig. 8. Data estimation and carrier phase estimation error effects on the coherent DiLL scheme. (a) Data estimation error. (b) Carrier phase estimation error.

signal power decreases by the factor of $\cos \left(\phi_{k}-\hat{\phi}_{k}\right)$ as in (6). The coherent DiLL has smaller DOA tracking error variance than the noncoherent DiLL, when the standard deviation is less than $12.5^{\circ}$ as shown in Fig. $8(\mathrm{~b})$.

\section{F. Initial DOA Tracking}

In this section, the number of iterations required for the initial DOA tracking stage is investigated. Fig. 9 shows the probability of the detection in the initial DOA tracking scheme with $N=8$ for the coherent and noncoherent DiLL scheme. In this simulation, we assume that the number of interfering signal sources are 9 which are uniformly distributed in $\left(-60^{\circ}, 60^{\circ}\right)$ and the desired user is at $60^{\circ}$. In Fig. 9, the vertical axis denotes the probability of the detection, $P_{\text {detection }}$ after iterations shown in horizontal axis when the initial DOA tracking value starts at $0^{\circ}$. Other parameters are the same as in Section VI-C. It is found that $P_{\text {detection }}$ for noncoherent DiLL is larger than that for the coherent DiLL because the locking range of the noncoherent

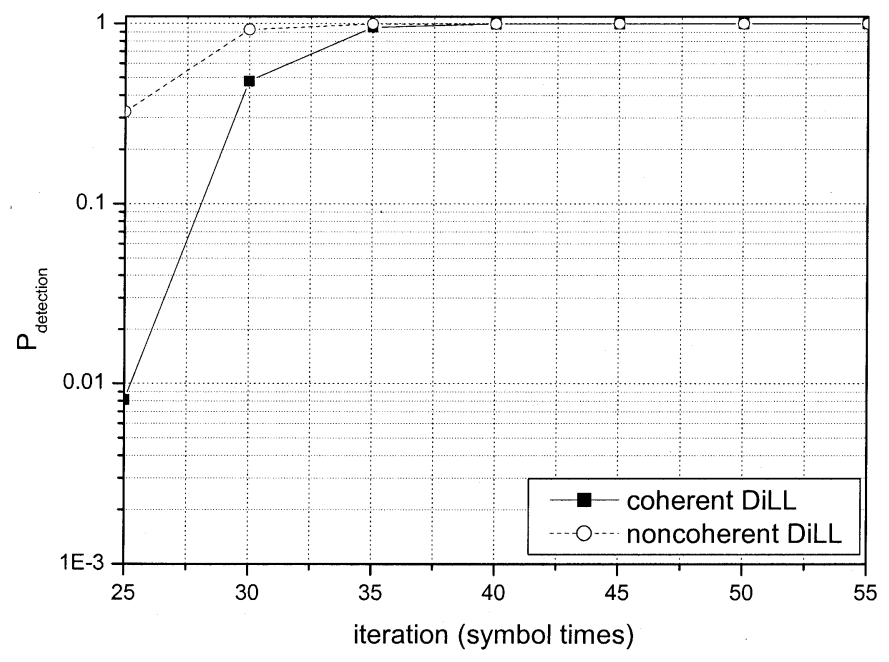

Fig. 9. Probability of the detection in the initial DOA tracking scheme.

DiLL is wider than that of the coherent DiLL. From Fig. 9, we may may observe that 43 and 48 iterations are enough for the initial tracking for the coherent and noncoherent DiLL scheme with the detection probability 0.99 in this simulation environments, respectively.

\section{CONCLUSION}

In this paper, a DOA tracking scheme, DiLL, has been proposed and appraised. It estimates the DOAs of signals iteratively by using the difference of the correlation between an input signal and the array response vectors whose directions are $\pm \Delta \theta$ shifted from the DOA tracking value. It has been implemented in both the coherent and noncoherent mode. Both DiLL schemes are conceptually simpler than the PASTd algorithm. The coherent DiLL scheme has better DOA tracking accuracy than the noncoherent scheme by a factor of more than 2 . The computational requirements of the DiLL scheme, which is $N K+\mathrm{O}(K)$ for coherent DiLL and $2 N K+\mathrm{O}(K)$ for noncoherent DiLL, is less than that of the PASTd, where $N$ is the number of antenna elements, and $K$ is the number of signal sources.

In the DiLL scheme, since the initial DOA tracking value should be in the locking range of the DOA of a signal, the initial DOA tracking scheme is proposed by using the noncoherent DiLL scheme with two antenna elements and the initial DOA tracking value, $0^{\circ}$.

\section{REFERENCES}

[1] L. C. Godara, "Application of antenna arrays to mobile communications, Part II: Beam-forming and direction-of-arrival consideration," Proc. IEEE, vol. 85, pp. 1195-1245, Aug. 1997.

[2] R. O. Schmidt, "Multiple emitter location and signal parameter estimation," IEEE Trans. Antennas Propagat., vol. 34, pp. 276-280, Mar. 1986.

[3] R. Roy and T. Kailath, "ESPRIT-Estimation of signal parameters via rotational invariance techniques," IEEE Trans. Acoust., Speech, Signal Processing, vol. 37, pp. 984-995, July 1989.

[4] Z. Yifeng, P. C. Yip, and H. Leung, "Tracking the direction-of-arrival of multiple moving targets by passive arrays: Algorithm," IEEE Trans. Signal Processing, vol. 47, pp. 2655-2666, Oct. 1999.

[5] B. Yang, "Projection approximation subspace tracking," IEEE Trans. Signal Processing, vol. 43, pp. 95-107, Jan. 1995.

[6] X. Wang and H. V. Poor, "Blind mutiuser detection: A subspace approach," IEEE Trans. Inform. Theory, vol. 44, pp. 677-690, Mar. 1998. 
[7] Z. Lei and T. J. Lim, "Estimation of directions of arrival of multipath signals in CDMA systems," IEEE Trans. Commun., vol. 48, pp. 1022-1028, June 2000.

[8] C. K. Sword, M. Simaan, and E. W. Kamen, "Multiple target angle tracking using sensor array output," IEEE Trans. Aerosp. Electron. Syst., vol. 26, pp. 367-372, Mar. 1990 .

[9] C. R. Sastry, E. W. Kamen, and M. Simaan, "An efficient algorithm for tracking the angles of arrival of moving targets," IEEE Trans. Signal Processing, vol. 39, pp. 242-246, Jan. 1991.

[10] C. R. Rao, L. Zhang, and L. C. Zhao, "Multiple target angle tracking using sensor array output," IEEE Trans. Aerosp. Electron. Syst., vol. 29, pp. 268-271, Jan. 1993.

[11] S. B. Park, C. S. Ryu, and K. K. Lee, "Multiple target tracking algorithm using predicted angles," IEEE Trans. Aerosp. Electron. Syst., vol. 30, pp. 643-648, Apr. 1994.

[12] C. R. Rao, C. R. Sastry, and B. Zhou, "Tracking the direction of arrival of multiple moving targets," IEEE Trans. Signal Processing, vol. 42, pp. 1133-1144, May 1995.

[13] A. Satish and R. Kashyap, "Multiple target tracking using maximum likelihood principle," IEEE Trans. Signal Processing, vol. 43, pp. 1677-1695, July 1995.

[14] Z. Yifeng, P. C. Yip, and H. Leung, "Tracking the direction-of-arrival of multiple moving targets by passive arrays: Asymptotic performance analysis," IEEE Trans. Signal Processing, vol. 47, pp. 2644-2654, Oct. 1999.

[15] H. Meyr, "Delay-lock tracking of stochastic signals," IEEE Trans. Commun., vol. 24, pp. 331-339, Mar. 1976.

[16] M. K. Simon, "Noncoherent pseudonoise code tracking performance of spread spectrum receivers," IEEE Trans. Commun., vol. 25, pp. 327-345, Mar. 1977.

[17] R. de Gaudenzi and M. Luise, "Decision-directed coherent delay-lock tracking loop for DS-spread-spectrum signals," IEEE Trans. Commun., vol. 39, pp. 758-765, May 1991.

[18] W. Hou and H. M. Kwon, "Interference suppression receiver with adaptive antenna array for code division multiple access communications systems," in Proc. IEEE Vehicular Technology Conf., Sept. 2000, pp. $1249-1254$

[19] - "Direction of arrival angle tracking algorithm for smart antennas," U.S. Patent 6483459 B1, Nov. 19, 2002.

[20] S. Min, K. B. Lee, and Y.-H. Lee, "'Direction of arrival' estimation algorithm: Direction lock loop," in Proc. CDMA Int. Conf., Nov. 2001

[21] T. Ihara, S. Tanaka, M. Sawahashi, and F. Adachi, "Fast two-step beam tracking algorithm of coherent adaptive antenna array diversity receiver in W-CDMA reverse link," IEICE Trans. Commun., vol. E84-B, pp. $1835-1848$, July 2001.

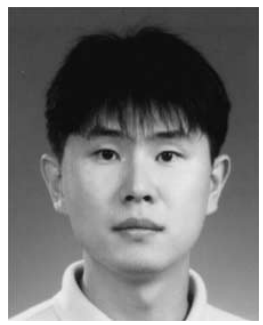

Seunghyun Min (S'96) received the B.S., M.S., and $\mathrm{Ph} . \mathrm{D}$. degrees in electrical engineering from Seoul National University, Seoul, Korea, in 1996, 1998, and 2003, respectively.

$\mathrm{He}$ is currently with the Telecommunication System Division, Samsung Electronics, Gyeonggi-do, Korea. His current research interests include mobile communications, spread-spectrum communication systems, smart antenna systems, and signal processing for communications.

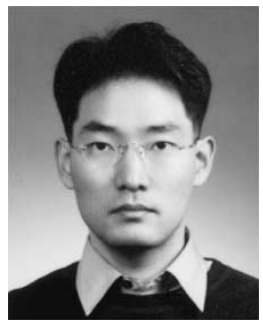

Dongyoun Seo was born in Seoul, Korea, in 1973. He received the B.S. and M.S. degrees in electrical engineering from Seoul National University, Seoul, Korea, in 1999 and 2001, respectively.

In 2002, he joined the Standardization and System Research Group, LG Electronics, Kyongki-do, Korea, where he has been involved in WCDMA physical layer standard of 3GPP TSG-RAN. His research interests include IMT-2000 standard, transmit diversity, and multiple-input multiple-output systems.

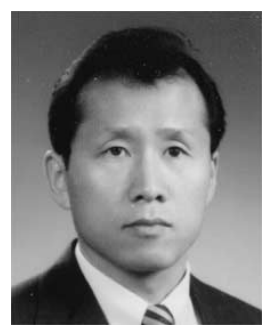

Kwang Bok (Ed) Lee (M'90) received the B.A.Sc. and M.Eng. degrees from the University of Toronto, Toronto, ON, Canada, in 1982 and 1986, respectively, and the Ph.D. degree from McMaster University, Hanilton, ON, Canada in 1990.

He was with Motorola Canada from 1982 to 1985 , and Motorola USA from 1990 to 1996, as a Senior Staff Engineer. At Motorola, he was involved in the research and development of wireless communication systems. He was with Bell-Northern Research, Canada, from 1989 to 1990. In March 1996, he joined the School of Electrical Engineering, Seoul National University, Seoul, Korea, where he is currently an Associate Professor. He was a Vice Chair of the School of Electrical Engineering from 2000 to 2002. He has been serving as a Consultant to a number of wireless industries. His research interests include mobile communications, communication theories, spread spectrum, and signal processing. He holds ten U.S. patents and two Korean patents, and has a number of patents pending.

Dr. Lee was an Editor of the IEEE Journal on SELECTED AREAS IN COMMUNICATIONS, Wireless Series, in 2001, and is currently the Editor of the IEEE TRANSACTIONS ON Wireless COMmunications. He received the Best Paper Award at the CDMA International Conference in 2000.

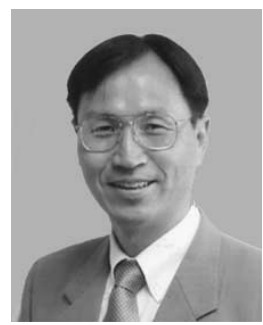

Hyuck M. Kwon (S'82-M'84-SM'96) was born in Korea in 1953. He received the B.S. and M.S. degrees in electrical engineering from Seoul National University, Seoul, Korea, in 1978 and 1980, respectively, and the Ph.D. degree in computer, information, and control engineering from the University of Michigan, Ann Arbor, in 1984

From 1985 to 1989 , he was with the University of Wisconsin, Milwaukee, as an Assistant Professor in the Electrical Engineering and Computer Science Department. From 1989 to 1993, he was with Lockheed Engineering and Sciences Company, Houston, TX, as a Principal Engineer, working for NASA space shuttle and space station satellite communication systems. Since 1993, he has been with the Department of Electrical and Computer Engineering, Wichita State University, Wichita, KS, where he is currently a Full Professor. In addition, he held several visiting and consulting positions with communication system industries, and a Visiting Associate Professor position at Texas A\&M University, College Station, in 1997. His current research interests are in wireless, code-division multiple-access (CDMA) spread spectrum, smart antennas, and multiple-antenna-array communication systems.

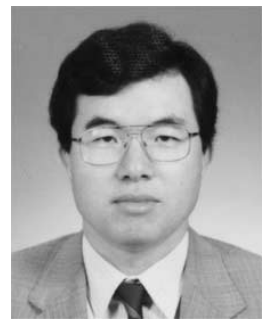

Yong-Hwan Lee (S'77-M'89) received the B.S. degree from Seoul National University, Seoul, Korea, in 1977, the M.S. degree from Korea Advanced Institute of Science and Technology (KAIST), Taejon, Korea, in 1980, and the Ph.D. degree from the University of Massachusetts, Amherst, in 1989, all in electrical engineering.

From 1980 to 1985, he was with the Korea Agency for Defense Development, where he was involved in development of shipboard weapon fire control systems. From 1989 to 1994, he was a Principal Engineer with Motorola, where he was engaged in research and development of data transmission systems including high-speed modems. In 1994, he joined the School of Electrical Engineering, Seoul National University, as a Faculty Member. His research areas are wired/wireless transmission systems including spread-spectrum systems, robust signal detection/estimation theory, and signal processing for communications. 